\title{
Polymer building blocks: self-assembly of silver(I)- cyclotriphosphazene cationic columns
}

\author{
Eric W. Ainscough,* Andrew M. Brodie,* Craig V. Depree, Geoffrey B. Jameson \\ and Carl A. Otter \\ Chemistry - Institute of Fundamental Sciences, Massey University, \\ Private Bag 11 222, Palmerston North, New Zealand
}

\section{Supporting Information}

\section{Experimental Section}

Analytical grade solvents were purchased from standard chemical suppliers and with the exception of tetrahydrofuran (THF), which was distilled from sodium/benzophenone, were used without further purification. Anhydrous $\mathrm{CuCl}_{2}$ (BDH) was dried at $110^{\circ} \mathrm{C}$ and $\mathrm{NaH}$ (Avocado) was obtained as a pure powder from a $60 \%$ dispersion in paraffin oil by washing with hexane. The cyclotriphosphazene, $\mathrm{N}_{3} \mathrm{P}_{3} \mathrm{Cl}_{6}$ (Aldrich) and 2-hydroxy-4-methylpyridine (Aldrich) were used as received. The ligands $\mathrm{L}$ and $\mathrm{MeL}$ were prepared by a literature methods ${ }^{1,2}$ as were $\left[\mathrm{Cu}\left(\mathrm{CH}_{3} \mathrm{CN}\right)_{4}\right] \mathrm{PF}_{6} \quad\left[\mathrm{Ag}\left(\mathrm{CH}_{3} \mathrm{CN}\right)_{4}\right] \mathrm{PF}_{6}{ }^{3}{ }^{3}$ Microanalyses were performed by the Campbell Microanalytical Laboratory, University of Otago. NMR spectra were recorded on a Jeol GX270W spectrometer, IR spectra were run as $\mathrm{KBr}$ discs on a Perkin-Elmer FT-IR Paragon 1000 spectrometer. Electrospray mass spectra were obtained from $\mathrm{CH}_{3} \mathrm{CN}$ solutions on a micromass $\mathrm{ZMD}$ spectrometer run in the positive ion mode. Listed peaks correspond to the most abundant isotopomer; assignments were made by a comparison of observed and simulated spectra.

\section{Syntheses}

$\left\{\left[\operatorname{AgL}_{\mathbf{P}} \mathbf{P F}_{6}\right\}_{\infty}\right.$ (1): The following procedure for the preparation of $\mathbf{1}$ is typical. $\left[\mathrm{Ag}\left(\mathrm{CH}_{3} \mathrm{CN}\right)_{4}\right] \mathrm{PF}_{6}(30 \mathrm{mg}, 0.14 \mathrm{mmol})$ and $\mathrm{L}(50 \mathrm{mg}, 0.14 \mathrm{mmol})$ dissolved in $\mathrm{CH}_{2} \mathrm{Cl}_{2}$ $(15 \mathrm{ml})$ were allowed to stir for $20 \mathrm{~min}$ at room temperature. Hexane was added to the 
solution to produce a precipitate that was filtered and dried under vacuum. Yield 52 mg. Found: 37.84, 2.44, 13.05\%. Required for $\mathrm{C}_{30} \mathrm{H}_{24} \mathrm{AgF}_{6} \mathrm{~N}_{9} \mathrm{O}_{6} \mathrm{P}_{4}: \mathrm{C}, 37.84 ; \mathrm{H}, 2.54$; N, 13.24\%. $v_{\max } / \mathrm{cm}^{-1}: 1224,1204,1184$ (ring PN). ${ }^{31} \mathrm{P} \mathrm{NMR}\left(\mathrm{CD}_{3} \mathrm{CN}\right): \delta 6.91(\mathrm{~s}, 3 \mathrm{P})$. $m / z$ (LSIMS): $808\left([\mathrm{AgL}]^{+}\right)$.

$\left\{[\mathbf{A g}(\mathrm{MeL})]_{\mathbf{P F}_{6}}\right\}_{\infty}$ (2): Found $\mathrm{C}, 41.58 ; \mathrm{H}, 3.32 ; \mathrm{N}, 11.96 \%$. Required for $\mathrm{C}_{36} \mathrm{H}_{36} \mathrm{AgF}_{6} \mathrm{~N}_{9} \mathrm{O}_{6} \mathrm{P}_{4}: \mathrm{C}, 41.72 ; \mathrm{H}, 3.50 ; \mathrm{N}, 12.16 \% . v_{\max } / \mathrm{cm}^{-1}: 1224,1211,1148$ (ring $\mathrm{PN}) .{ }^{31} \mathrm{P}$ NMR $\left(\mathrm{CD}_{3} \mathrm{CN}\right): \delta 6.89(\mathrm{~s}, 3 \mathrm{P}) . \mathrm{m} / \mathrm{z}(\mathrm{LSIMS}): 892\left([\mathrm{Ag}(\mathrm{MeL})]^{+}\right)$.

$\left\{[\mathrm{Ag}(\mathrm{MeL})] \mathrm{NO}_{3}\right\}_{\infty}(3):$ A solution of $\mathrm{AgNO}_{3}(11 \mathrm{mg})$ in $\mathrm{MeOH}(3 \mathrm{~mL})$ was layered onto a solution of $\mathrm{MeL}(50 \mathrm{mg})$ in $\mathrm{CH}_{2} \mathrm{Cl}_{2}(3 \mathrm{~mL})$ and then the mixture was allowed to evaporate to $c a .2 \mathrm{~mL}$. A small crop of crystals was collected for microanalysis and Xray diffraction. Analytical data fitted the presence of 0.5 solvent molecules although the X-ray structure performed on a crystal which was taken directly from the mother liquor indicated the presence of approximately 1.33 molecules per formula unit. Found: C, 43.93; H, 3.71; N, 14.19\%. Required for $\mathrm{C}_{36} \mathrm{H}_{36} \mathrm{AgN}_{10} \mathrm{O}_{9} \mathrm{P}_{3} \cdot 0.5 \mathrm{CH}_{2} \mathrm{Cl}_{2}$ : C, 43.98; H, 3.71; N, 14.06\%. $v_{\max } / \mathrm{cm}^{-1}: 1223,1147$ (ring $\left.\mathrm{PN}\right) .{ }^{31} \mathrm{P} \mathrm{NMR}\left(\mathrm{CD}_{3} \mathrm{CN}\right): \delta$ $6.89(\mathrm{~s}, 3 \mathrm{P}) . \mathrm{m} / \mathrm{z}$ (ESMS): $892\left([\mathrm{Ag}(\mathrm{MeL})]^{+}\right)$. A further crop of the product was obtained by taking the mother liquor to dryness and allowing diethylether vapour to diffuse into an acetonitrile solution of the residue.

$\left\{[\mathbf{C u L}]_{P F}\right\}_{\infty}$ (4): Found: 39.62, 2.65, 13.72\%. Required for $\mathrm{C}_{30} \mathrm{H}_{24} \mathrm{CuF}_{6} \mathrm{~N}_{9} \mathrm{O}_{6} \mathrm{P}_{4}: \mathrm{C}$, 39.68; H, 2.66; N, 13.88\%. $v_{\max } / \mathrm{cm}^{-1}: 1226,1189$ (ring $\left.\mathrm{PN}\right) .{ }^{31} \mathrm{P} \mathrm{NMR}\left(\mathrm{CD}_{3} \mathrm{CN}\right): \delta$ 6.48 (s, 3P). $m / z$ (LSIMS): $762\left([\mathrm{CuL}]^{+}\right)$. A similar reaction using MeL instead of L afforded a colourless oil which turned green in acetonitrile/ether solution over several days. Green crystals, which were obtained in low yield, were identified as $\left[\mathrm{Cu}_{2}(\mathrm{MeL})_{2}(\mu-\mathrm{OH})(\mu-4-\mathrm{MeOpy})\right]\left(\mathrm{PF}_{6}\right)_{2}$ (4-MepyO is the 4-methyl-2-pyridyloxylato anion) (5) by single crystal X-ray crystallography.

\section{X-ray Crystallographic Structure Determinations of 1, 3 and 5}

The X-ray data was collected on a Siemens P4 four circle diffractometer, using a Siemens SMART 1K CCD area detector. The crystals were mounted in an inert oil, transferred into the cold gas stream of the detector and irradiated with graphite 
monochromated Mo-K $\mathrm{K}_{\alpha}(\lambda=0.71073 \AA)$ X-rays. The data were collected by the SMART program and processed with SAINT to apply Lorentz and polarisation corrections to the diffraction spots (integrated 3 dimensionally). Crystal data are given below. The structures were solved by direct methods and refined using the SHELXTL program. ${ }^{4}$ Hydrogen atoms were calculated at ideal positions. For the solution of $\mathbf{3}$, the electron density of disordered $\mathrm{CH}_{2} \mathrm{Cl}_{2}$ was removed from the unit cell using PLATON/SQUEEZE. ${ }^{5}$ Approximately 1.33 molecules of $\mathrm{CH}_{2} \mathrm{Cl}_{2}$ per cell were removed ( $255 \mathrm{e}^{-}$per cell and $449.4 .8 \AA^{3}$ was left by the void). The solution of 5 revealed the presence of two acetonitrile molecules (one of which was disordered) in the crystal lattice. Atomic coordinates, equivalent isotropic displacement parameters, bond lengths and angles and anisotropic parameters for all 3 structures are given in the Appendix.

\section{Crystallographic data}

$\left\{[\mathbf{A g L}] \mathbf{P F}_{6}\right\}_{\infty}$ (1): Chemical formula $\mathrm{C}_{30} \mathrm{H}_{24} \mathrm{AgF}_{6} \mathrm{~N}_{9} \mathrm{O}_{6} \mathrm{P}_{4} ; M=952.33$, orthorhombic, space group Pbca, $a=15.20880(10), b=18.5883(3), c=25.7347(2) \AA, U=$ $7275.35(15) \AA^{3}, T=203(2) \mathrm{K}, Z=8, D_{\mathrm{c}}=1.739 \mathrm{gcm}^{-3}, F(000)=3808, \mu(\mathrm{Mo}-\mathrm{K})=$ $1.797 \mathrm{~mm}^{-1}, 41771$ reflections measured $\left(3.16<2 \theta<54.98^{\circ}\right), 8154$ unique $\left(R_{\text {int }}=\right.$ 0.0209). Refinement of 505 parameters converged at $R_{1}=0.0290$ [observed data: 7217 $\left.\left|F_{\mathrm{o}}\right|>4 \sigma\left(F_{\mathrm{o}}\right)\right] . w R\left(F^{2}\right)=0.0800$ (all data).

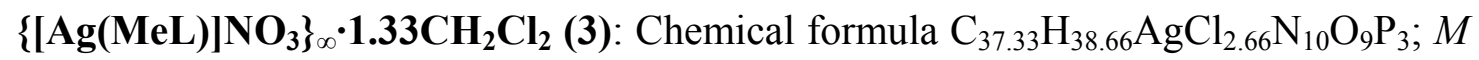
$=1066.49$, monoclinic, space group $P 2(1) / n, a=18.82520(10), b=10.7023(2), c=$ 21.6663(3) $\AA, \beta=91.2680(10) . U=4354.10(10) \AA^{3}, T=84(2) \mathrm{K}, Z=4, D_{\mathrm{c}}=1.623$ $\mathrm{gcm}^{-3}, F(000)=2199, \mu(\mathrm{Mo}-\mathrm{K})=0.801 \mathrm{~mm}^{-1}, 8909$ reflections measured $(2.84<2 \theta<$ $52.81^{\circ}$ ), 8909 unique. Refinement of 587 parameters converged at $R_{1}=0.0540$ [observed data: $\left.6400\left|F_{\mathrm{o}}\right|>4 \sigma\left(F_{\mathrm{o}}\right)\right] . w R\left(F^{2}\right)=0.1602$ (all data).

$\left[\mathrm{Cu}_{2}(\mathrm{MeL})_{2}(\mu-\mathrm{OH})(\mu-4-\mathrm{MeOpy})\right]\left(\mathrm{PF}_{6}\right)_{2} \cdot \mathbf{2 C H}_{3} \mathrm{CN} \quad$ (5): Chemical formula $\mathrm{C}_{82} \mathrm{H}_{85} \mathrm{Cu}_{2} \mathrm{~F}_{12} \mathrm{~N}_{21} \mathrm{O}_{14} \mathrm{P}_{8} ; \quad M=2191.57$, monoclinic, space group $P 2(1) / c, a=$ 19.1972(2), $b=33.2535(4), c=16.5053(2) \AA, \beta=114.9320(10), U=9554.64(19) \AA^{3}$, $T=84(2) \mathrm{K}, Z=4, D_{\mathrm{c}}=1.528 \mathrm{gcm}^{-3}, F(000)=4488, \mu(\mathrm{Mo}-\mathrm{K})=0.725 \mathrm{~mm}^{-1}, 42654$ reflections measured $\left(5.44<2 \theta<47^{\circ}\right), 13681$ unique $\left(R_{\text {int }}=0.1507\right)$. Refinement of 
1314 parameters converged at $R_{1}=0.0838$ [observed data: $\left.7298\left|F_{\mathrm{o}}\right|>4 \sigma\left(F_{\mathrm{o}}\right)\right] . w R\left(F^{2}\right)$ $=0.1754$ (all data).

\section{References}

(1) Cho, Y.; Baek, H.; Sohn, Y. S. Macromolecules, 1999, 32, 2167-2172.

(2) Ainscough, E. W.; Brodie, A. M.; Depree, C. V.; Moubaraki, B.; Murray, K. S.; Otter, C. A. Dalton Trans., accepted for publication (B508620E).

(3) Kubas, G. J. Inorg. Synth., 1990, 28, 68-70.

(4) Sheldrick, G. M. SHELXL Suite of Programs for Crystal Structure Analysis, Institüt für Anorganische Chemie der Universität, Tammanstrasse 4, Göttingen, Germany, 1998.

(5) van der Sluis, P.; Spek, A. L. Acta Cryst., 1990, A46, 194-201. 


\section{APPENDIX}

Table 1 Atomic coordinates and equivalent isotropic displacement parameters for $\left\{[\mathrm{AgL}] \mathrm{PF}_{6}\right\}_{\infty}(\mathbf{1})$.

Table 2 Bond lengths and angles for $\left\{\left[\mathrm{AgL}_{\mathrm{P}} \mathrm{PF}_{6}\right\}_{\infty}(\mathbf{1})\right.$.

Table 3 Anisotropic displacement parameters for $\left\{\left[\mathrm{AgL}_{\mathrm{P}} \mathrm{PF}_{6}\right\}_{\infty}(\mathbf{1})\right.$.

Table 4 Atomic coordinates and equivalent isotropic displacement parameters for $\left\{[\mathrm{Ag}(\mathrm{MeL})] \mathrm{NO}_{3}\right\}_{\infty} \cdot 1.33 \mathrm{CH}_{2} \mathrm{Cl}_{2}(\mathbf{3})$

Table 5 Bond lengths and angles for $\left\{[\mathrm{Ag}(\mathrm{MeL})] \mathrm{NO}_{3}\right\}_{\infty} \cdot 1.33 \mathrm{CH}_{2} \mathrm{Cl}_{2}(\mathbf{3})$

Table 6 Anisotropic displacement parameters for $\left\{[\mathrm{Ag}(\mathrm{MeL})] \mathrm{NO}_{3}\right\}_{\infty} \cdot 1.33 \mathrm{CH}_{2} \mathrm{Cl}_{2}$ (3)

Table 7 Atomic coordinates and equivalent isotropic displacement parameters for $\left[\mathrm{Cu}_{2}(\mathrm{MeL})_{2}(\mu-\mathrm{OH})(\mu-4-\mathrm{MeOpy})\right]\left(\mathrm{PF}_{6}\right)_{2} \cdot 2 \mathrm{CH}_{3} \mathrm{CN}(\mathbf{5})$

Table 8 Bond lengths and angles for $\left[\mathrm{Cu}_{2}(\mathrm{MeL})_{2}(\pi-\mathrm{OH})(\pi-4-\mathrm{MeOpy})\right]\left(\mathrm{PF}_{6}\right)_{2} \cdot 2 \mathrm{CH}_{3} \mathrm{CN}(\mathbf{5})$

Table 9 Anisotropic displacement parameters for $\left[\mathrm{Cu}_{2}(\mathrm{MeL})_{2}((\pi-\mathrm{OH})(\mu-4-\mathrm{MeOpy})]\left(\mathrm{PF}_{6}\right)_{2} \cdot 2 \mathrm{CH}_{3} \mathrm{CN}(\mathbf{5})\right.$ 
Table 1. Atomic coordinates $\left(x 10^{4}\right)$ and equivalent isotropic displacement parameters $\left(\AA^{2} \times 10^{3}\right)$ for $\left\{[A g L] \mathrm{PF}_{6}\right\}_{\infty}$ (1).

$\mathrm{U}(\mathrm{eq})$ is defined as one third of the trace of the orthogonalized $\mathrm{U}^{i j}$ tensor.

\begin{tabular}{|c|c|c|c|c|}
\hline & $x$ & $\mathrm{Y}$ & z & $\mathrm{U}(\mathrm{eq})$ \\
\hline $\mathrm{Ag}(1)$ & $7604(1)$ & $2133(1)$ & $5155(1)$ & $33(1)$ \\
\hline$P(1)$ & $8898(1)$ & $3557(1)$ & $5018(1)$ & $25(1)$ \\
\hline$P(3)$ & $10622(1)$ & $3687(1)$ & $4724(1)$ & $25(1)$ \\
\hline$P(2)$ & $10002(1)$ & $2382(1)$ & $5101(1)$ & $22(1)$ \\
\hline $\mathrm{N}(3)$ & $9703(1)$ & $4032(1)$ & $4852(1)$ & $30(1)$ \\
\hline$N(2)$ & 10765 (1) & $2875(1)$ & $4879(1)$ & $25(1)$ \\
\hline $\mathrm{N}(1)$ & $9050(1)$ & $2732(1)$ & $5156(1)$ & $25(1)$ \\
\hline$O(1)$ & $8469(1)$ & $3984(1)$ & $5483(1)$ & $35(1)$ \\
\hline$O(2)$ & $8113(1)$ & $3615(1)$ & $4602(1)$ & $31(1)$ \\
\hline$O(5)$ & $11391(1)$ & $4170(1)$ & $4955(1)$ & $29(1)$ \\
\hline$O(6)$ & $10786(1)$ & $3828(1)$ & $4124(1)$ & $34(1)$ \\
\hline$O(3)$ & $10299(1)$ & $2006(1)$ & $5628(1)$ & $27(1)$ \\
\hline$O(4)$ & $9988(1)$ & $1703(1)$ & $4731(1)$ & $27(1)$ \\
\hline$N(16)$ & $7280(1)$ & $3264(1)$ & $5714(1)$ & $30(1)$ \\
\hline $\mathrm{N}(26)$ & $8977(1)$ & $3073(1)$ & $3973(1)$ & $39(1)$ \\
\hline$N(36)$ & $10165(1)$ & $3046(1)$ & $6116(1)$ & $32(1)$ \\
\hline$N(46)$ & $8973(1)$ & $1015(1)$ & $5192(1)$ & $30(1)$ \\
\hline C (11) & $7845(1)$ & $3771(1)$ & $5841(1)$ & $30(1)$ \\
\hline C (12) & $7856(2)$ & $4146(2)$ & $6301(1)$ & $52(1)$ \\
\hline C (13) & $7207(2)$ & 3991 (2) & $6657(1)$ & $70(1)$ \\
\hline C (14) & $6584(2)$ & $3474(2)$ & $6535(1)$ & $54(1)$ \\
\hline C (15) & $6645(1)$ & 3125 (1) & $6067(1)$ & $35(1)$ \\
\hline C (21) & $8217(1)$ & $3364(1)$ & $4099(1)$ & $33(1)$ \\
\hline C (22) & $7504(2)$ & $3438(2)$ & $3768(1)$ & $49(1)$ \\
\hline C (23) & $7605(2)$ & $3188(2)$ & $3270(1)$ & $64(1)$ \\
\hline C (24) & $8398(2)$ & $2887(2)$ & $3120(1)$ & $61(1)$ \\
\hline C (25) & $9059(2)$ & $2839(2)$ & $3481(1)$ & $50(1)$ \\
\hline C (51) & $11674(1)$ & $4120(1)$ & $5468(1)$ & $27(1)$ \\
\hline C (52) & $11374(2)$ & $4611(1)$ & $5827(1)$ & $36(1)$ \\
\hline C (53) & $11733(2)$ & $4578(1)$ & $6319(1)$ & $46(1)$ \\
\hline C ( 54$)$ & $12369(2)$ & $4063(2)$ & $6426(1)$ & $47(1)$ \\
\hline C ( 55) & $12612(2)$ & $3594(1)$ & $6039(1)$ & $40(1)$ \\
\hline$C(61)$ & $11541(1)$ & $3782(1)$ & $3827(1)$ & $27(1)$ \\
\hline$C(62)$ & 11455 (2) & $4049(1)$ & $3328(1)$ & $37(1)$ \\
\hline C (63) & $12194(2)$ & $4040(1)$ & $3016(1)$ & $46(1)$ \\
\hline$C(64)$ & $12970(2)$ & $3758(1)$ & $3211(1)$ & $45(1)$ \\
\hline$C(65)$ & $12975(2)$ & $3496(1)$ & $3710(1)$ & $41(1)$ \\
\hline C (31) & $10231(1)$ & $2339(1)$ & $6111(1)$ & $25(1)$ \\
\hline C (32) & $10243(1)$ & $1892(1)$ & $6540(1)$ & $31(1)$ \\
\hline C (33) & $10172(2)$ & $2219(1)$ & $7019(1)$ & $40(1)$ \\
\hline C (34) & $10086(2)$ & $2958(1)$ & $7047(1)$ & $44(1)$ \\
\hline C (35) & $10090(2)$ & $3348(1)$ & $6590(1)$ & $39(1)$ \\
\hline C ( 41$)$ & $9488(1)$ & $1078(1)$ & $4786(1)$ & $25(1)$ \\
\hline C ( 42) & $9592(1)$ & $578(1)$ & $4392(1)$ & $33(1)$ \\
\hline C ( 43) & $9122(2)$ & $-54(1)$ & $4440(1)$ & $42(1)$ \\
\hline C ( 44$)$ & $8579(2)$ & $-153(1)$ & $4866(1)$ & $40(1)$ \\
\hline C ( 45) & $8520(1)$ & $389(1)$ & $5227(1)$ & $35(1)$ \\
\hline$P(4)$ & $9796(1)$ & $134(1)$ & $2814(1)$ & $38(1)$ \\
\hline$F(1)$ & $8943(1)$ & $-239(1)$ & $3058(1)$ & $66(1)$ \\
\hline$F(2)$ & $10351(1)$ & $-101(1)$ & $3313(1)$ & $49(1)$ \\
\hline$F(3)$ & $9237(1)$ & $365(1)$ & $2314(1)$ & $59(1)$ \\
\hline$F(4)$ & $10047(1)$ & $-612(1)$ & $2538(1)$ & $60(1)$ \\
\hline$F(5)$ & $9548(1)$ & $877(1)$ & $3089(1)$ & $51(1)$ \\
\hline $\mathrm{F}(6)$ & $10649(1)$ & $503(1)$ & $2567(1)$ & $52(1)$ \\
\hline $\mathrm{N}(56)$ & $12271(1)$ & $3612(1)$ & $5555(1)$ & $31(1)$ \\
\hline$N(66)$ & $12263(1)$ & $3504(1)$ & $4024(1)$ & $33(1)$ \\
\hline
\end{tabular}


Table 2. Bond lengths $[\AA]$ and angles $\left[{ }^{\circ}\right]$ for $\left\{[A g L] \mathrm{PF}_{6}\right\}_{\infty}$ (1).

\begin{tabular}{|c|c|}
\hline $\mathrm{Ag}(1)-\mathrm{N}(56) \# 1$ & $2.3474(18)$ \\
\hline $\operatorname{Ag}(1)-N(1)$ & $2.4643(16)$ \\
\hline $\mathrm{Ag}(1)-\mathrm{N}(66) \# 1$ & $2.4773(17)$ \\
\hline $\operatorname{Ag}(1)-N(16)$ & $2.5952(17)$ \\
\hline$P(1)-N(3)$ & $1.5703(17)$ \\
\hline$P(1)-O(1)$ & $1.5771(15)$ \\
\hline $\mathrm{P}(1)-\mathrm{N}(1)$ & $1.5913(16)$ \\
\hline$P(1)-O(2)$ & $1.6079(15)$ \\
\hline$P(3)-N(3)$ & $1.5725(17)$ \\
\hline $\mathrm{P}(3)-\mathrm{N}(2)$ & $1.5772(16)$ \\
\hline$P(3)-O(6)$ & $1.5864(15)$ \\
\hline$P(3)-O(5)$ & $1.5890(14)$ \\
\hline$P(2)-O(4)$ & $1.5803(13)$ \\
\hline$P(2)-N(2)$ & $1.5856(16)$ \\
\hline$P(2)-O(3)$ & $1.5920(14)$ \\
\hline$P(2)-N(1)$ & $1.5932(16)$ \\
\hline $\mathrm{O}(1)-\mathrm{C}(11)$ & $1.379(2)$ \\
\hline $\mathrm{O}(2)-\mathrm{C}(21)$ & $1.383(3)$ \\
\hline$O(5)-C(51)$ & $1.392(2)$ \\
\hline$O(6)-C(61)$ & $1.382(2)$ \\
\hline$O(3)-C(31)$ & $1.392(2)$ \\
\hline$O(4)-C(41)$ & $1.397(2)$ \\
\hline$N(16)-C(11)$ & $1.317(3)$ \\
\hline$N(16)-C(15)$ & $1.352(3)$ \\
\hline$N(26)-C(21)$ & $1.316(3)$ \\
\hline$N(26)-C(25)$ & $1.345(3)$ \\
\hline$N(36)-C(31)$ & $1.318(2)$ \\
\hline$N(36)-C(35)$ & $1.346(3)$ \\
\hline$N(46)-C(41)$ & $1.312(3)$ \\
\hline$N(46)-C(45)$ & $1.354(3)$ \\
\hline$C(11)-C(12)$ & $1.376(3)$ \\
\hline$C(12)-C(13)$ & $1.377(4)$ \\
\hline $\mathrm{C}(12)-\mathrm{H}(12)$ & 0.9400 \\
\hline$C(13)-C(14)$ & $1.385(4)$ \\
\hline $\mathrm{C}(13)-\mathrm{H}(13)$ & 0.9400 \\
\hline$C(14)-C(15)$ & $1.371(3)$ \\
\hline $\mathrm{C}(14)-\mathrm{H}(14)$ & 0.9400 \\
\hline $\mathrm{C}(15)-\mathrm{H}(15)$ & 0.9400 \\
\hline$C(21)-C(22)$ & $1.387(3)$ \\
\hline$C(22)-C(23)$ & $1.374(4)$ \\
\hline $\mathrm{C}(22)-\mathrm{H}(22)$ & 0.9400 \\
\hline$C(23)-C(24)$ & $1.384(4)$ \\
\hline $\mathrm{C}(23)-\mathrm{H}(23)$ & 0.9400 \\
\hline$C(24)-C(25)$ & $1.372(4)$ \\
\hline $\mathrm{C}(24)-\mathrm{H}(24)$ & 0.9400 \\
\hline $\mathrm{C}(25)-\mathrm{H}(25)$ & 0.9400 \\
\hline$C(51)-N(56)$ & $1.328(3)$ \\
\hline$C(51)-C(52)$ & $1.377(3)$ \\
\hline$C(52)-C(53)$ & $1.380(3)$ \\
\hline $\mathrm{C}(52)-\mathrm{H}(52)$ & 0.9400 \\
\hline$C(53)-C(54)$ & $1.388(4)$ \\
\hline $\mathrm{C}(53)-\mathrm{H}(53)$ & 0.9400 \\
\hline$C(54)-C(55)$ & $1.374(4)$ \\
\hline $\mathrm{C}(54)-\mathrm{H}(54)$ & 0.9400 \\
\hline$C(55)-N(56)$ & $1.351(3)$ \\
\hline $\mathrm{C}(55)-\mathrm{H}(55)$ & 0.9400 \\
\hline $\mathrm{C}(61)-\mathrm{N}(66)$ & $1.314(3)$ \\
\hline$C(61)-C(62)$ & $1.384(3)$ \\
\hline$C(62)-C(63)$ & $1.380(3)$ \\
\hline $\mathrm{C}(62)-\mathrm{H}(62)$ & 0.9400 \\
\hline$C(63)-C(64)$ & $1.385(4)$ \\
\hline $\mathrm{C}(63)-\mathrm{H}(63)$ & 0.9400 \\
\hline$C(64)-C(65)$ & $1.374(3)$ \\
\hline $\mathrm{C}(64)-\mathrm{H}(64)$ & 0.9400 \\
\hline
\end{tabular}




\begin{tabular}{|c|c|}
\hline $\mathrm{C}(65)-\mathrm{N}(66)$ & $1.352(3)$ \\
\hline $\mathrm{C}(65)-\mathrm{H}(65)$ & 0.9400 \\
\hline$C(31)-C(32)$ & $1.381(3)$ \\
\hline$C(32)-C(33)$ & $1.380(3)$ \\
\hline $\mathrm{C}(32)-\mathrm{H}(32)$ & 0.9400 \\
\hline$C(33)-C(34)$ & $1.382(3)$ \\
\hline $\mathrm{C}(33)-\mathrm{H}(33)$ & 0.9400 \\
\hline$C(34)-C(35)$ & $1.382(3)$ \\
\hline $\mathrm{C}(34)-\mathrm{H}(34)$ & 0.9400 \\
\hline $\mathrm{C}(35)-\mathrm{H}(35)$ & 0.9400 \\
\hline$C(41)-C(42)$ & $1.384(3)$ \\
\hline$C(42)-C(43)$ & $1.380(3)$ \\
\hline $\mathrm{C}(42)-\mathrm{H}(42)$ & 0.9400 \\
\hline$C(43)-C(44)$ & $1.385(3)$ \\
\hline $\mathrm{C}(43)-\mathrm{H}(43)$ & 0.9400 \\
\hline$C(44)-C(45)$ & $1.372(3)$ \\
\hline $\mathrm{C}(44)-\mathrm{H}(44)$ & 0.9400 \\
\hline $\mathrm{C}(45)-\mathrm{H}(45)$ & 0.9400 \\
\hline$P(4)-F(5)$ & $1.5970(15)$ \\
\hline$P(4)-F(2)$ & $1.5978(14)$ \\
\hline$P(4)-F(1)$ & $1.5990(17)$ \\
\hline$P(4)-F(6)$ & $1.5999(16)$ \\
\hline$P(4)-F(3)$ & $1.6004(15)$ \\
\hline$P(4)-F(4)$ & $1.6046(16)$ \\
\hline $\mathrm{N}(56)-\mathrm{Ag}(1) \# 2$ & $2.3474(18)$ \\
\hline$N(66)-A g(1) \# 2$ & $2.4773(17)$ \\
\hline $\mathrm{N}(56) \# 1-\mathrm{Ag}(1)-\mathrm{N}(1)$ & $117.43(5)$ \\
\hline $\mathrm{N}(56) \# 1-\mathrm{Ag}(1)-\mathrm{N}(66) \# 1$ & $109.66(6)$ \\
\hline $\mathrm{N}(1)-\mathrm{Ag}(1)-\mathrm{N}(66) \# 1$ & $113.65(6)$ \\
\hline $\mathrm{N}(56) \# 1-\mathrm{Ag}(1)-\mathrm{N}(16)$ & $150.26(6)$ \\
\hline$N(1)-\operatorname{Ag}(1)-N(16)$ & $78.62(5)$ \\
\hline $\mathrm{N}(66) \# 1-\mathrm{Ag}(1)-\mathrm{N}(16)$ & $82.78(6)$ \\
\hline$N(3)-P(1)-O(1)$ & $104.27(9)$ \\
\hline $\mathrm{N}(3)-\mathrm{P}(1)-\mathrm{N}(1)$ & $119.37(9)$ \\
\hline $\mathrm{O}(1)-\mathrm{P}(1)-\mathrm{N}(1)$ & $112.06(8)$ \\
\hline$N(3)-P(1)-O(2)$ & $111.04(9)$ \\
\hline $\mathrm{O}(1)-\mathrm{P}(1)-\mathrm{O}(2)$ & $99.50(8)$ \\
\hline$N(1)-P(1)-O(2)$ & $108.75(8)$ \\
\hline$N(3)-P(3)-N(2)$ & $117.42(9)$ \\
\hline$N(3)-P(3)-O(6)$ & $105.98(9)$ \\
\hline$N(2)-P(3)-O(6)$ & $112.46(9)$ \\
\hline$N(3)-P(3)-O(5)$ & $110.19(9)$ \\
\hline$N(2)-P(3)-O(5)$ & $110.19(8)$ \\
\hline$O(6)-P(3)-O(5)$ & $98.97(8)$ \\
\hline $\mathrm{O}(4)-\mathrm{P}(2)-\mathrm{N}(2)$ & $104.68(8)$ \\
\hline$O(4)-P(2)-O(3)$ & $99.60(7)$ \\
\hline $\mathrm{N}(2)-\mathrm{P}(2)-\mathrm{O}(3)$ & $110.62(8)$ \\
\hline $\mathrm{O}(4)-\mathrm{P}(2)-\mathrm{N}(1)$ & $111.61(8)$ \\
\hline $\mathrm{N}(2)-\mathrm{P}(2)-\mathrm{N}(1)$ & $117.53(9)$ \\
\hline $\mathrm{O}(3)-\mathrm{P}(2)-\mathrm{N}(1)$ & $111.14(8)$ \\
\hline$P(1)-N(3)-P(3)$ & $121.37(11)$ \\
\hline$P(3)-N(2)-P(2)$ & $122.90(10)$ \\
\hline$P(1)-N(1)-P(2)$ & $120.33(10)$ \\
\hline $\mathrm{P}(1)-\mathrm{N}(1)-\mathrm{Ag}(1)$ & $107.78(8)$ \\
\hline $\mathrm{P}(2)-\mathrm{N}(1)-\mathrm{Ag}(1)$ & $128.76(8)$ \\
\hline$C(11)-O(1)-P(1)$ & $130.25(13)$ \\
\hline$C(21)-O(2)-P(1)$ & $121.04(12)$ \\
\hline$C(51)-O(5)-P(3)$ & $122.99(12)$ \\
\hline$C(61)-O(6)-P(3)$ & $131.12(12)$ \\
\hline$C(31)-O(3)-P(2)$ & $123.01(12)$ \\
\hline $\mathrm{C}(41)-\mathrm{O}(4)-\mathrm{P}(2)$ & $127.62(12)$ \\
\hline $\mathrm{C}(11)-\mathrm{N}(16)-\mathrm{C}(15)$ & $115.94(18)$ \\
\hline $\mathrm{C}(11)-\mathrm{N}(16)-\mathrm{Ag}(1)$ & $126.34(13)$ \\
\hline $\mathrm{C}(15)-\mathrm{N}(16)-\mathrm{Ag}(1)$ & $110.69(14)$ \\
\hline $\mathrm{C}(21)-\mathrm{N}(26)-\mathrm{C}(25)$ & $116.6(2)$ \\
\hline $\mathrm{C}(31)-\mathrm{N}(36)-\mathrm{C}(35)$ & $115.56(18)$ \\
\hline $\mathrm{C}(41)-\mathrm{N}(46)-\mathrm{C}(45)$ & $115.71(18)$ \\
\hline
\end{tabular}




\begin{tabular}{|c|c|}
\hline $\mathrm{N}(16)-\mathrm{C}(11)-\mathrm{C}(12)$ & $125.7(2)$ \\
\hline $\mathrm{N}(16)-\mathrm{C}(11)-\mathrm{O}(1)$ & $119.31(18)$ \\
\hline $\mathrm{C}(12)-\mathrm{C}(11)-\mathrm{O}(1)$ & $114.92(19)$ \\
\hline$C(11)-C(12)-C(13)$ & $117.2(2)$ \\
\hline $\mathrm{C}(11)-\mathrm{C}(12)-\mathrm{H}(12)$ & 121.4 \\
\hline $\mathrm{C}(13)-\mathrm{C}(12)-\mathrm{H}(12)$ & 121.4 \\
\hline$C(12)-C(13)-C(14)$ & $119.1(2)$ \\
\hline $\mathrm{C}(12)-\mathrm{C}(13)-\mathrm{H}(13)$ & 120.5 \\
\hline $\mathrm{C}(14)-\mathrm{C}(13)-\mathrm{H}(13)$ & 120.5 \\
\hline$C(15)-C(14)-C(13)$ & $118.8(2)$ \\
\hline $\mathrm{C}(15)-\mathrm{C}(14)-\mathrm{H}(14)$ & 120.6 \\
\hline $\mathrm{C}(13)-\mathrm{C}(14)-\mathrm{H}(14)$ & 120.6 \\
\hline $\mathrm{N}(16)-\mathrm{C}(15)-\mathrm{C}(14)$ & $123.2(2)$ \\
\hline $\mathrm{N}(16)-\mathrm{C}(15)-\mathrm{H}(15)$ & 118.4 \\
\hline $\mathrm{C}(14)-\mathrm{C}(15)-\mathrm{H}(15)$ & 118.4 \\
\hline $\mathrm{N}(26)-\mathrm{C}(21)-\mathrm{O}(2)$ & $118.03(18)$ \\
\hline$N(26)-C(21)-C(22)$ & $125.1(2)$ \\
\hline $\mathrm{O}(2)-\mathrm{C}(21)-\mathrm{C}(22)$ & $116.8(2)$ \\
\hline$C(23)-C(22)-C(21)$ & $116.9(2)$ \\
\hline$C(23)-C(22)-H(22)$ & 121.5 \\
\hline $\mathrm{C}(21)-\mathrm{C}(22)-\mathrm{H}(22)$ & 121.5 \\
\hline$C(22)-C(23)-C(24)$ & $119.7(3)$ \\
\hline $\mathrm{C}(22)-\mathrm{C}(23)-\mathrm{H}(23)$ & 120.2 \\
\hline $\mathrm{C}(24)-\mathrm{C}(23)-\mathrm{H}(23)$ & 120.2 \\
\hline$C(25)-C(24)-C(23)$ & $118.4(3)$ \\
\hline $\mathrm{C}(25)-\mathrm{C}(24)-\mathrm{H}(24)$ & 120.8 \\
\hline $\mathrm{C}(23)-\mathrm{C}(24)-\mathrm{H}(24)$ & 120.8 \\
\hline $\mathrm{N}(26)-\mathrm{C}(25)-\mathrm{C}(24)$ & $123.3(3)$ \\
\hline $\mathrm{N}(26)-\mathrm{C}(25)-\mathrm{H}(25)$ & 118.4 \\
\hline $\mathrm{C}(24)-\mathrm{C}(25)-\mathrm{H}(25)$ & 118.4 \\
\hline$N(56)-C(51)-C(52)$ & $125.7(2)$ \\
\hline $\mathrm{N}(56)-\mathrm{C}(51)-\mathrm{O}(5)$ & $114.78(17)$ \\
\hline$C(52)-C(51)-O(5)$ & $119.33(18)$ \\
\hline$C(51)-C(52)-C(53)$ & $117.1(2)$ \\
\hline $\mathrm{C}(51)-\mathrm{C}(52)-\mathrm{H}(52)$ & 121.4 \\
\hline$C(53)-C(52)-H(52)$ & 121.4 \\
\hline$C(52)-C(53)-C(54)$ & $119.2(2)$ \\
\hline$C(52)-C(53)-H(53)$ & 120.4 \\
\hline$C(54)-C(53)-H(53)$ & 120.4 \\
\hline$C(55)-C(54)-C(53)$ & $118.8(2)$ \\
\hline$C(55)-C(54)-H(54)$ & 120.6 \\
\hline $\mathrm{C}(53)-\mathrm{C}(54)-\mathrm{H}(54)$ & 120.6 \\
\hline$N(56)-C(55)-C(54)$ & $123.3(2)$ \\
\hline $\mathrm{N}(56)-\mathrm{C}(55)-\mathrm{H}(55)$ & 118.3 \\
\hline$C(54)-C(55)-H(55)$ & 118.3 \\
\hline$N(66)-C(61)-O(6)$ & $120.37(17)$ \\
\hline $\mathrm{N}(66)-\mathrm{C}(61)-\mathrm{C}(62)$ & $125.3(2)$ \\
\hline $\mathrm{O}(6)-\mathrm{C}(61)-\mathrm{C}(62)$ & $114.33(18)$ \\
\hline$C(63)-C(62)-C(61)$ & $117.3(2)$ \\
\hline $\mathrm{C}(63)-\mathrm{C}(62)-\mathrm{H}(62)$ & 121.4 \\
\hline $\mathrm{C}(61)-\mathrm{C}(62)-\mathrm{H}(62)$ & 121.4 \\
\hline$C(62)-C(63)-C(64)$ & $119.3(2)$ \\
\hline $\mathrm{C}(62)-\mathrm{C}(63)-\mathrm{H}(63)$ & 120.4 \\
\hline $\mathrm{C}(64)-\mathrm{C}(63)-\mathrm{H}(63)$ & 120.4 \\
\hline$C(65)-C(64)-C(63)$ & $118.5(2)$ \\
\hline $\mathrm{C}(65)-\mathrm{C}(64)-\mathrm{H}(64)$ & 120.8 \\
\hline $\mathrm{C}(63)-\mathrm{C}(64)-\mathrm{H}(64)$ & 120.8 \\
\hline $\mathrm{N}(66)-\mathrm{C}(65)-\mathrm{C}(64)$ & $123.5(2)$ \\
\hline $\mathrm{N}(66)-\mathrm{C}(65)-\mathrm{H}(65)$ & 118.3 \\
\hline $\mathrm{C}(64)-\mathrm{C}(65)-\mathrm{H}(65)$ & 118.3 \\
\hline $\mathrm{N}(36)-\mathrm{C}(31)-\mathrm{C}(32)$ & $126.36(18)$ \\
\hline $\mathrm{N}(36)-\mathrm{C}(31)-\mathrm{O}(3)$ & $117.24(17)$ \\
\hline$C(32)-C(31)-O(3)$ & $116.41(17)$ \\
\hline$C(33)-C(32)-C(31)$ & $116.62(19)$ \\
\hline $\mathrm{C}(33)-\mathrm{C}(32)-\mathrm{H}(32)$ & 121.7 \\
\hline $\mathrm{C}(31)-\mathrm{C}(32)-\mathrm{H}(32)$ & 121.7 \\
\hline$C(32)-C(33)-C(34)$ & $119.4(2)$ \\
\hline $\mathrm{C}(32)-\mathrm{C}(33)-\mathrm{H}(33)$ & 120.3 \\
\hline
\end{tabular}




\begin{tabular}{|c|c|}
\hline $\mathrm{C}(34)-\mathrm{C}(33)-\mathrm{H}(33)$ & 120.3 \\
\hline$C(35)-C(34)-C(33)$ & $118.5(2)$ \\
\hline $\mathrm{C}(35)-\mathrm{C}(34)-\mathrm{H}(34)$ & 120.8 \\
\hline $\mathrm{C}(33)-\mathrm{C}(34)-\mathrm{H}(34)$ & 120.8 \\
\hline $\mathrm{N}(36)-\mathrm{C}(35)-\mathrm{C}(34)$ & $123.5(2)$ \\
\hline $\mathrm{N}(36)-\mathrm{C}(35)-\mathrm{H}(35)$ & 118.2 \\
\hline $\mathrm{C}(34)-\mathrm{C}(35)-\mathrm{H}(35)$ & 118.2 \\
\hline$N(46)-C(41)-C(42)$ & $126.29(18)$ \\
\hline $\mathrm{N}(46)-\mathrm{C}(41)-\mathrm{O}(4)$ & $118.73(16)$ \\
\hline$C(42)-C(41)-O(4)$ & $114.98(17)$ \\
\hline$C(43)-C(42)-C(41)$ & $116.4(2)$ \\
\hline$C(43)-C(42)-H(42)$ & 121.8 \\
\hline $\mathrm{C}(41)-\mathrm{C}(42)-\mathrm{H}(42)$ & 121.8 \\
\hline$C(42)-C(43)-C(44)$ & $119.6(2)$ \\
\hline $\mathrm{C}(42)-\mathrm{C}(43)-\mathrm{H}(43)$ & 120.2 \\
\hline $\mathrm{C}(44)-\mathrm{C}(43)-\mathrm{H}(43)$ & 120.2 \\
\hline$C(45)-C(44)-C(43)$ & $118.5(2)$ \\
\hline $\mathrm{C}(45)-\mathrm{C}(44)-\mathrm{H}(44)$ & 120.8 \\
\hline $\mathrm{C}(43)-\mathrm{C}(44)-\mathrm{H}(44)$ & 120.8 \\
\hline$N(46)-C(45)-C(44)$ & $123.5(2)$ \\
\hline $\mathrm{N}(46)-\mathrm{C}(45)-\mathrm{H}(45)$ & 118.3 \\
\hline $\mathrm{C}(44)-\mathrm{C}(45)-\mathrm{H}(45)$ & 118.3 \\
\hline$F(5)-P(4)-F(2)$ & $90.28(8)$ \\
\hline$F(5)-P(4)-F(1)$ & $90.56(10)$ \\
\hline$F(2)-P(4)-F(1)$ & $89.72(9)$ \\
\hline$F(5)-P(4)-F(6)$ & $89.82(9)$ \\
\hline$F(2)-P(4)-F(6)$ & $90.44(9)$ \\
\hline$F(1)-P(4)-F(6)$ & $179.59(11)$ \\
\hline$F(5)-P(4)-F(3)$ & $89.96(8)$ \\
\hline$F(2)-P(4)-F(3)$ & $179.69(9)$ \\
\hline$F(1)-P(4)-F(3)$ & $90.08(10)$ \\
\hline$F(6)-P(4)-F(3)$ & $89.76(9)$ \\
\hline$F(5)-P(4)-F(4)$ & $179.84(12)$ \\
\hline$F(2)-P(4)-F(4)$ & $89.67(8)$ \\
\hline$F(1)-P(4)-F(4)$ & $89.60(10)$ \\
\hline$F(6)-P(4)-F(4)$ & $90.03(10)$ \\
\hline$F(3)-P(4)-F(4)$ & $90.10(8)$ \\
\hline$C(51)-N(56)-C(55)$ & $115.82(19)$ \\
\hline $\mathrm{C}(51)-\mathrm{N}(56)-\mathrm{Ag}(1) \# 2$ & $115.83(13)$ \\
\hline $\mathrm{C}(55)-\mathrm{N}(56)-\mathrm{Ag}(1) \# 2$ & $128.35(15)$ \\
\hline$C(61)-N(66)-C(65)$ & $116.25(18)$ \\
\hline $\mathrm{C}(61)-\mathrm{N}(66)-\mathrm{Ag}(1) \# 2$ & $133.77(14)$ \\
\hline $\mathrm{C}(65)-\mathrm{N}(66)-\mathrm{Ag}(1) \# 2$ & $109.72(14)$ \\
\hline
\end{tabular}

Symmetry transformations used to generate equivalent atoms: $\# 1 \mathrm{x}-1 / 2,-\mathrm{y}+1 / 2,-\mathrm{z}+1, \# 2 \mathrm{x}+1 / 2,-\mathrm{y}+1 / 2,-\mathrm{z}+1$ 
Table 3. Anisotropic displacement parameters $\left(\AA^{2} \times 10^{3}\right)$ for $\left\{[\mathrm{AgL}] \mathrm{PF}_{6}\right\}_{\infty}$ (1) . The anisotropic displacement factor exponent takes the form: $-2 \pi^{2}\left[h^{2} a *^{2} U^{11}+\ldots+2 h k a * b * U^{12}\right]$.

\begin{tabular}{|c|c|c|c|c|c|c|}
\hline & $\mathrm{U}^{11}$ & $\mathrm{U}^{22}$ & $\mathrm{U}^{33}$ & $\mathrm{U}^{23}$ & $\mathrm{U}^{13}$ & $\mathrm{U}^{12}$ \\
\hline $\operatorname{Ag}(1)$ & $31(1)$ & $34(1)$ & $34(1)$ & $0(1)$ & $4(1)$ & $-9(1)$ \\
\hline $\mathrm{P}(1)$ & $21(1)$ & $21(1)$ & $34(1)$ & $2(1)$ & $3(1)$ & $1(1)$ \\
\hline$P(3)$ & $20(1)$ & $24(1)$ & $30(1)$ & $7(1)$ & $-1(1)$ & $-1(1)$ \\
\hline$P(2)$ & $23(1)$ & $19(1)$ & $24(1)$ & $0(1)$ & $1(1)$ & $0(1)$ \\
\hline$N(3)$ & $23(1)$ & $22(1)$ & $46(1)$ & $9(1)$ & $3(1)$ & $1(1)$ \\
\hline$N(2)$ & $21(1)$ & $22(1)$ & $31(1)$ & $1(1)$ & $1(1)$ & $1(1)$ \\
\hline$N(1)$ & $22(1)$ & $21(1)$ & $31(1)$ & $2(1)$ & $2(1)$ & $0(1)$ \\
\hline$O(1)$ & $34(1)$ & $24(1)$ & $46(1)$ & $-4(1)$ & $11(1)$ & $-1(1)$ \\
\hline$O(2)$ & $22(1)$ & $34(1)$ & $39(1)$ & $4(1)$ & $1(1)$ & $5(1)$ \\
\hline$O(5)$ & $25(1)$ & $25(1)$ & $36(1)$ & $8(1)$ & $-3(1)$ & $-4(1)$ \\
\hline$O(6)$ & $22(1)$ & $50(1)$ & $32(1)$ & $14(1)$ & $-2(1)$ & $-1(1)$ \\
\hline$O(3)$ & $36(1)$ & $21(1)$ & $25(1)$ & $0(1)$ & $-1(1)$ & $5(1)$ \\
\hline$O(4)$ & $31(1)$ & $22(1)$ & $29(1)$ & $-3(1)$ & $4(1)$ & $-3(1)$ \\
\hline $\mathrm{N}(16)$ & $26(1)$ & $32(1)$ & $32(1)$ & $-4(1)$ & $1(1)$ & $2(1)$ \\
\hline$N(26)$ & $28(1)$ & $52(1)$ & $37(1)$ & $5(1)$ & $4(1)$ & $1(1)$ \\
\hline $\mathrm{N}(36)$ & $41(1)$ & $23(1)$ & $32(1)$ & $-1(1)$ & $2(1)$ & $-1(1)$ \\
\hline$N(46)$ & $30(1)$ & $25(1)$ & $35(1)$ & $0(1)$ & $5(1)$ & $1(1)$ \\
\hline C (11) & $23(1)$ & $28(1)$ & $41(1)$ & $-6(1)$ & $4(1)$ & $6(1)$ \\
\hline C (12) & $42(1)$ & $57(2)$ & $58(2)$ & $-29(1)$ & $12(1)$ & $-11(1)$ \\
\hline C (13) & $65(2)$ & $88(2)$ & $58(2)$ & $-43(2)$ & $24(2)$ & $-20(2)$ \\
\hline C (14) & $45(1)$ & $66(2)$ & $50(2)$ & $-19(1)$ & $22(1)$ & $-6(1)$ \\
\hline C (15) & $27(1)$ & $39(1)$ & $40(1)$ & $-5(1)$ & $3(1)$ & $1(1)$ \\
\hline C (21) & $26(1)$ & $35(1)$ & $37(1)$ & $11(1)$ & $0(1)$ & $-2(1)$ \\
\hline C (22) & $32(1)$ & $68(2)$ & $47(1)$ & $10(1)$ & $-8(1)$ & $4(1)$ \\
\hline C (23) & $49(2)$ & $101(3)$ & $43(2)$ & $12(2)$ & $-13(1)$ & $-4(2)$ \\
\hline C (24) & $54(2)$ & $97(2)$ & $32(1)$ & $8(1)$ & $4(1)$ & $-13(2)$ \\
\hline C (25) & $38(1)$ & $75(2)$ & $39(1)$ & $2(1)$ & $10(1)$ & $-3(1)$ \\
\hline C (51) & $24(1)$ & $24(1)$ & $34(1)$ & $5(1)$ & $-1(1)$ & $-6(1)$ \\
\hline C (52) & $38(1)$ & $26(1)$ & $46(1)$ & $1(1)$ & $4(1)$ & $-2(1)$ \\
\hline C (53) & $56(2)$ & $38(1)$ & $43(1)$ & $-7(1)$ & $6(1)$ & $-6(1)$ \\
\hline C (54) & $53(2)$ & $55(2)$ & $35(1)$ & $1(1)$ & $-8(1)$ & $-8(1)$ \\
\hline C (55) & $38(1)$ & $44(1)$ & $40(1)$ & $6(1)$ & $-9(1)$ & $0(1)$ \\
\hline C (61) & $26(1)$ & $28(1)$ & $28(1)$ & $4(1)$ & $-2(1)$ & $-6(1)$ \\
\hline C (62) & $39(1)$ & $41(1)$ & $31(1)$ & $6(1)$ & $-4(1)$ & $4(1)$ \\
\hline C (63) & $55(1)$ & $54(2)$ & $29(1)$ & $9(1)$ & $5(1)$ & $3(1)$ \\
\hline C (64) & $40(1)$ & $58(2)$ & $37(1)$ & $-2(1)$ & $8(1)$ & $-1(1)$ \\
\hline C (65) & $29(1)$ & $58(2)$ & $36(1)$ & $0(1)$ & $0(1)$ & $1(1)$ \\
\hline C (31) & $23(1)$ & $25(1)$ & $26(1)$ & $-1(1)$ & $0(1)$ & $0(1)$ \\
\hline C (32) & $34(1)$ & $26(1)$ & $32(1)$ & $3(1)$ & $0(1)$ & $1(1)$ \\
\hline C (33) & 51 (1) & $39(1)$ & $29(1)$ & $3(1)$ & $0(1)$ & $-1(1)$ \\
\hline C (34) & $59(2)$ & $41(1)$ & $31(1)$ & $-9(1)$ & $3(1)$ & $-5(1)$ \\
\hline C (35) & $53(1)$ & $26(1)$ & $39(1)$ & $-8(1)$ & $4(1)$ & $-4(1)$ \\
\hline C (41) & $25(1)$ & $20(1)$ & $31(1)$ & $1(1)$ & $-4(1)$ & $1(1)$ \\
\hline C (42) & $37(1)$ & $30(1)$ & $31(1)$ & $-7(1)$ & $1(1)$ & $-2(1)$ \\
\hline C (43) & $47(1)$ & $31(1)$ & $49(1)$ & $-12(1)$ & $-3(1)$ & $-6(1)$ \\
\hline C (44) & $35(1)$ & $25(1)$ & $60(2)$ & $-1(1)$ & $-2(1)$ & $-6(1)$ \\
\hline C (45) & $30(1)$ & $29(1)$ & $47(1)$ & $6(1)$ & $5(1)$ & $-1(1)$ \\
\hline P (4) & $50(1)$ & $28(1)$ & $35(1)$ & $3(1)$ & $-12(1)$ & $-3(1)$ \\
\hline$F(1)$ & $59(1)$ & $58(1)$ & $83(1)$ & $23(1)$ & $-6(1)$ & $-16(1)$ \\
\hline$F(2)$ & $64(1)$ & $48(1)$ & $36(1)$ & $2(1)$ & $-16(1)$ & $7(1)$ \\
\hline F (3) & $81(1)$ & $41(1)$ & $53(1)$ & $9(1)$ & $-35(1)$ & $-6(1)$ \\
\hline$F(4)$ & $95(1)$ & $34(1)$ & 51 (1) & $-10(1)$ & $-24(1)$ & $8(1)$ \\
\hline F (5) & 65 (1) & $38(1)$ & $50(1)$ & $-4(1)$ & $-3(1)$ & $6(1)$ \\
\hline$F(6)$ & $58(1)$ & $55(1)$ & $44(1)$ & $0(1)$ & $1(1)$ & $-9(1)$ \\
\hline N (5 6) & $26(1)$ & $31(1)$ & $36(1)$ & $4(1)$ & $-3(1)$ & $-1(1)$ \\
\hline$N(66)$ & $27(1)$ & $43(1)$ & $30(1)$ & $6(1)$ & $-2(1)$ & $-2(1)$ \\
\hline
\end{tabular}


Table 4. Atomic coordinates $\left(x 10^{4}\right)$ and equivalent isotropic displacement parameters $\left(\AA^{2} \times 10^{3}\right)$ for $\left\{[\mathrm{Ag}(\mathrm{MeL})] \mathrm{NO}_{3}\right\}_{\infty} \cdot 1 \cdot 33 \mathrm{CH}_{2} \mathrm{Cl}_{2}$ (3) .

$\mathrm{U}(\mathrm{eq})$ is defined as one third of the trace of the orthogonalized $\mathrm{U}^{i j}$ tensor.

\begin{tabular}{|c|c|c|c|c|}
\hline & $\mathrm{x}$ & Y & $z$ & $\mathrm{U}(\mathrm{eq})$ \\
\hline$N(36)$ & $7349(2)$ & $9983(3)$ & $3607(2)$ & $22(1)$ \\
\hline$N(46)$ & $8730(2)$ & $10014(3)$ & $2165(2)$ & $23(1)$ \\
\hline$C(45)$ & $9283(2)$ & $10317(5)$ & $1818(2)$ & $31(1)$ \\
\hline $\operatorname{Ag}(1)$ & $6817(1)$ & $6064(1)$ & $2055(1)$ & $26(1)$ \\
\hline$C(31)$ & $6819(2)$ & $9199(4)$ & $3511(2)$ & $22(1)$ \\
\hline C (32) & $6326(3)$ & $8857(4)$ & $3946(2)$ & $30(1)$ \\
\hline C (33) & $6398(3)$ & $9347(5)$ & $4535(2)$ & $36(1)$ \\
\hline$C(34)$ & $6962(3)$ & $10161(5)$ & $4653(2)$ & $37(1)$ \\
\hline$C(37)$ & $5896(3)$ & $9019(6)$ & $5030(3)$ & $53(2)$ \\
\hline$C(22)$ & $6907(3)$ & $8458(5)$ & $-429(2)$ & $33(1)$ \\
\hline$C(27)$ & $7221(4)$ & $8865(8)$ & $-1543(3)$ & $71(2)$ \\
\hline$C(25)$ & $5542(3)$ & $9213(5)$ & $-615(2)$ & $35(1)$ \\
\hline C (23) & $6696(3)$ & $8852(5)$ & $-1012(2)$ & $44(1)$ \\
\hline$C(24)$ & $5991(3)$ & $9235(5)$ & $-1099(2)$ & $41(1)$ \\
\hline $\mathrm{C}(21)$ & $6405(3)$ & $8490(4)$ & $31(2)$ & $26(1)$ \\
\hline C (13) & $4549(3)$ & $5203(4)$ & $700(2)$ & $34(1)$ \\
\hline$C(12)$ & $4726(2)$ & $6456(4)$ & $806(2)$ & $30(1)$ \\
\hline$C(17)$ & $3858(3)$ & $4826(5)$ & $401(3)$ & $52(2)$ \\
\hline$C(14)$ & $5053(3)$ & $4302(4)$ & $879(2)$ & $35(1)$ \\
\hline$C(15)$ & $5682(3)$ & $4670(4)$ & $1150(2)$ & $40(1)$ \\
\hline$C(11)$ & $5362(2)$ & $6721(4)$ & $1097(2)$ & $24(1)$ \\
\hline$C(41)$ & $8422(2)$ & $8917(4)$ & $2051(2)$ & $21(1)$ \\
\hline$C(51)$ & $5407(2)$ & $11539(5)$ & $2296(2)$ & $33(1)$ \\
\hline C (43) & $9220(3)$ & $8394(5)$ & $1258(2)$ & $33(1)$ \\
\hline$C(42)$ & $8635(2)$ & $8081(4)$ & $1602(2)$ & $28(1)$ \\
\hline$C(47)$ & $9487(3)$ & $7493(6)$ & $786(3)$ & $54(2)$ \\
\hline C (35) & $7414(3)$ & $10457(4)$ & $4186(2)$ & $30(1)$ \\
\hline$C(44)$ & $9535(3)$ & $9530(5)$ & $1363(2)$ & $34(1)$ \\
\hline $\mathrm{N}(1)$ & $6748(2)$ & $8139(3)$ & $1791(2)$ & $21(1)$ \\
\hline$N(26)$ & $5741(2)$ & $8827(3)$ & $-35(2)$ & $30(1)$ \\
\hline$N(16)$ & $5844(2)$ & 5885 (3) & $1268(2)$ & $31(1)$ \\
\hline $\mathrm{N}(3)$ & $6136(2)$ & $10068(3)$ & $1196(2)$ & $23(1)$ \\
\hline $\mathrm{N}(2)$ & $7043(2)$ & $10490(3)$ & $2167(2)$ & $20(1)$ \\
\hline$O(2)$ & $6633(2)$ & $8102(3)$ & $627(1)$ & $25(1)$ \\
\hline$O(1)$ & $5501(2)$ & 7975 (3) & $1241(1)$ & $24(1)$ \\
\hline$O(6)$ & $6912(2)$ & $12059(2)$ & $1265(1)$ & $23(1)$ \\
\hline$O(5)$ & $5953(2)$ & $11967(3)$ & $1929(1)$ & $24(1)$ \\
\hline$O(3)$ & $6733(2)$ & $8628(3)$ & $2937(1)$ & $22(1)$ \\
\hline$O(4)$ & $7875(2)$ & $8612(3)$ & $2441(1)$ & $20(1)$ \\
\hline$N(53 A)$ & $4203(8)$ & $7425(14)$ & $3995(8)$ & $48(2)$ \\
\hline$O(52 A)$ & $3724(12)$ & $7910(30)$ & $4234(15)$ & $134(5)$ \\
\hline$O(50 A)$ & $4762(6)$ & $7072(13)$ & $4209(6)$ & $89(4)$ \\
\hline$O(51 A)$ & $4164(9)$ & $7211(18)$ & $3418(7)$ & $147(7)$ \\
\hline$N(53 B)$ & $4060(10)$ & $7243(19)$ & $3967(9)$ & $48(2)$ \\
\hline$O(52 B)$ & $3614(14)$ & $7700(40)$ & $4258(19)$ & $134(5)$ \\
\hline$O(50 B)$ & $4642(9)$ & $7631(14)$ & $3855(9)$ & $101(6)$ \\
\hline$O(51 B)$ & $3942(10)$ & $6179(16)$ & $3723(10)$ & $144(9)$ \\
\hline$P(3)$ & $6518(1)$ & $11029(1)$ & $1649(1)$ & $18(1)$ \\
\hline$P(2)$ & $7078(1)$ & $9036(1)$ & $2302(1)$ & $18(1)$ \\
\hline $\mathrm{P}(1)$ & $6262(1)$ & $8621(1)$ & $1231(1)$ & $20(1)$ \\
\hline C ( 52) & $4884(3)$ & $12386(6)$ & $2445(3)$ & $52(2)$ \\
\hline C (53) & $4345(3)$ & $11986(11)$ & $2807(4)$ & $81(3)$ \\
\hline C ( 54$)$ & $4350(4)$ & $10751(11)$ & $3006(3)$ & $86(3)$ \\
\hline C ( 55) & $4886(3)$ & $9982(9)$ & $2827(3)$ & $72(2)$ \\
\hline$N(56)$ & $5426(2)$ & $10356(5)$ & $2464(2)$ & $44(1)$ \\
\hline C ( 57$)$ & $3774(4)$ & $12887(12)$ & $3011(5)$ & $138(5)$ \\
\hline$C(61 A)$ & $7200(5)$ & $11869(7)$ & $672(4)$ & $47(2)$ \\
\hline
\end{tabular}




\begin{tabular}{lllrl}
$\mathrm{C}(62 \mathrm{~A})$ & $7822(3)$ & $11171(5)$ & $608(3)$ & $31(2)$ \\
$\mathrm{C}(63 \mathrm{~A})$ & $8092(4)$ & $11005(6)$ & $26(4)$ & $32(2)$ \\
$\mathrm{C}(64 \mathrm{~A})$ & $7713(3)$ & $11560(6)$ & $-447(4)$ & $41(2)$ \\
$\mathrm{C}(65 \mathrm{~A})$ & $7084(4)$ & $12252(7)$ & $-339(4)$ & $50(2)$ \\
$\mathrm{N}(66 \mathrm{~A})$ & $6826(3)$ & $12404(5)$ & $225(3)$ & $38(2)$ \\
$\mathrm{C}(67 \mathrm{~A})$ & $8746(4)$ & $10281(8)$ & $-78(4)$ & $67(3)$ \\
$\mathrm{C}(61 \mathrm{~B})$ & $7265(5)$ & $11672(9)$ & $725(4)$ & $47(2)$ \\
$\mathrm{C}(62 \mathrm{~B})$ & $7087(5)$ & $12217(9)$ & $159(5)$ & $31(2)$ \\
$\mathrm{C}(63 \mathrm{~B})$ & $7436(6)$ & $11835(10)$ & $-363(5)$ & $32(2)$ \\
$\mathrm{C}(64 \mathrm{~B})$ & $7941(5)$ & $10927(10)$ & $-271(5)$ & $41(2)$ \\
$\mathrm{C}(65 \mathrm{~B})$ & $8088(6)$ & $10418(11)$ & $324(5)$ & $50(2)$ \\
$\mathrm{N}(66 \mathrm{~B})$ & $7749(5)$ & $10790(10)$ & $825(4)$ & $38(2)$ \\
$\mathrm{C}(67 \mathrm{~B})$ & $7272(8)$ & $12374(14)$ & $-977(5)$ & $67(3)$ \\
\hline
\end{tabular}


Table 5. Bond lengths $[\AA]$ and angles $\left[{ }^{\circ}\right]$ for $\left\{[\mathrm{Ag}(\mathrm{MeL})] \mathrm{NO}_{3}\right\}_{\infty} \cdot 1.33 \mathrm{CH}_{2} \mathrm{Cl}_{2}$ (3).

\begin{tabular}{|c|c|}
\hline $\mathrm{N}(36)-\mathrm{Ag}(1) \# 1$ & $2.440(4)$ \\
\hline $\mathrm{N}(46)-\operatorname{Ag}(1) \# 1$ & $2.293(4)$ \\
\hline $\operatorname{Ag}(1)-N(46) \# 2$ & $2.293(4)$ \\
\hline $\operatorname{Ag}(1)-N(1)$ & $2.296(3)$ \\
\hline $\operatorname{Ag}(1)-N(36) \# 2$ & $2.440(4)$ \\
\hline $\operatorname{Ag}(1)-N(16)$ & $2.482(4)$ \\
\hline$N(1)-P(2)$ & $1.583(4)$ \\
\hline $\mathrm{N}(1)-\mathrm{P}(1)$ & $1.588(4)$ \\
\hline $\mathrm{N}(3)-\mathrm{P}(1)$ & $1.569(3)$ \\
\hline$N(3)-P(3)$ & $1.582(4)$ \\
\hline $\mathrm{N}(2)-\mathrm{P}(2)$ & $1.585(3)$ \\
\hline$N(2)-P(3)$ & $1.588(3)$ \\
\hline $\mathrm{O}(2)-\mathrm{P}(1)$ & $1.598(3)$ \\
\hline $\mathrm{O}(1)-\mathrm{P}(1)$ & $1.590(3)$ \\
\hline$O(6)-P(3)$ & $1.575(3)$ \\
\hline$O(5)-P(3)$ & $1.592(3)$ \\
\hline$O(3)-P(2)$ & $1.594(3)$ \\
\hline$O(4)-P(2)$ & $1.590(3)$ \\
\hline$N(46)-C(41)$ & $1.331(5)$ \\
\hline$N(46)-C(45)$ & $1.338(6)$ \\
\hline$C(45)-C(44)$ & $1.389(7)$ \\
\hline$N(36)-C(31)$ & $1.317(5)$ \\
\hline $\mathrm{N}(36)-\mathrm{C}(35)$ & $1.357(5)$ \\
\hline$C(31)-C(32)$ & $1.386(6)$ \\
\hline$C(31)-O(3)$ & $1.393(5)$ \\
\hline$C(32)-C(33)$ & $1.383(7)$ \\
\hline $\mathrm{C}(32)-\mathrm{H}(32)$ & 0.9300 \\
\hline$C(33)-C(34)$ & $1.392(7)$ \\
\hline C (33) -C (37) & $1.487(7)$ \\
\hline$C(34)-C(35)$ & $1.373(7)$ \\
\hline $\mathrm{C}(34)-\mathrm{H}(34)$ & 0.9300 \\
\hline $\mathrm{C}(37)-\mathrm{H}(37 \mathrm{~A})$ & 0.9600 \\
\hline$C(37)-H(37 B)$ & 0.9600 \\
\hline $\mathrm{C}(37)-\mathrm{H}(37 \mathrm{C})$ & 0.9600 \\
\hline$C(22)-C(23)$ & $1.381(7)$ \\
\hline$C(22)-C(21)$ & $1.389(7)$ \\
\hline $\mathrm{C}(22)-\mathrm{H}(22)$ & 0.9300 \\
\hline$C(27)-C(23)$ & $1.534(8)$ \\
\hline$C(27)-H(27 A)$ & 0.9600 \\
\hline $\mathrm{C}(27)-\mathrm{H}(27 \mathrm{~B})$ & 0.9600 \\
\hline $\mathrm{C}(27)-\mathrm{H}(27 \mathrm{C})$ & 0.9600 \\
\hline$C(25)-C(24)$ & $1.362(8)$ \\
\hline $\mathrm{C}(25)-\mathrm{N}(26)$ & $1.367(6)$ \\
\hline $\mathrm{C}(25)-\mathrm{H}(25)$ & 0.9300 \\
\hline$C(23)-C(24)$ & $1.399(8)$ \\
\hline $\mathrm{C}(24)-\mathrm{H}(24)$ & 0.9300 \\
\hline $\mathrm{C}(21)-\mathrm{N}(26)$ & $1.307(6)$ \\
\hline $\mathrm{C}(21)-\mathrm{O}(2)$ & $1.412(5)$ \\
\hline$C(13)-C(12)$ & $1.400(6)$ \\
\hline C (13) -C (14) & $1.402(7)$ \\
\hline$C(13)-C(17)$ & $1.496(7)$ \\
\hline$C(12)-C(11)$ & $1.370(6)$ \\
\hline $\mathrm{C}(12)-\mathrm{H}(12)$ & 0.9300 \\
\hline $\mathrm{C}(17)-\mathrm{H}(17 \mathrm{~A})$ & 0.9600 \\
\hline $\mathrm{C}(17)-\mathrm{H}(17 \mathrm{~B})$ & 0.9600 \\
\hline $\mathrm{C}(17)-\mathrm{H}(17 \mathrm{C})$ & 0.9600 \\
\hline$C(14)-C(15)$ & $1.368(7)$ \\
\hline $\mathrm{C}(14)-\mathrm{H}(14)$ & 0.9300 \\
\hline $\mathrm{C}(15)-\mathrm{N}(16)$ & $1.359(6)$ \\
\hline $\mathrm{C}(15)-\mathrm{H}(15)$ & 0.9300 \\
\hline $\mathrm{C}(11)-\mathrm{N}(16)$ & $1.322(6)$ \\
\hline C (11) - O (1) & $1.401(5)$ \\
\hline $\mathrm{C}(41)-\mathrm{O}(4)$ & $1.385(5)$ \\
\hline
\end{tabular}




\begin{tabular}{|c|c|}
\hline$C(41)-C(42)$ & $1.388(6)$ \\
\hline$C(51)-N(56)$ & $1.317(7)$ \\
\hline$C(51)-C(52)$ & $1.382(7)$ \\
\hline$C(51)-O(5)$ & $1.390(6)$ \\
\hline$C(43)-C(44)$ & $1.370(7)$ \\
\hline$C(43)-C(42)$ & $1.384(7)$ \\
\hline$C(43)-C(47)$ & $1.501(7)$ \\
\hline$O(6)-C(61 B)$ & $1.421(7)$ \\
\hline$O(6)-C(61 A)$ & $1.421(7)$ \\
\hline $\mathrm{C}(42)-\mathrm{H}(42)$ & 0.9300 \\
\hline $\mathrm{C}(47)-\mathrm{H}(47 \mathrm{~A})$ & 0.9600 \\
\hline $\mathrm{C}(47)-\mathrm{H}(47 \mathrm{~B})$ & 0.9600 \\
\hline $\mathrm{C}(47)-\mathrm{H}(47 \mathrm{C})$ & 0.9600 \\
\hline $\mathrm{C}(35)-\mathrm{H}(35)$ & 0.9300 \\
\hline $\mathrm{C}(44)-\mathrm{H}(44)$ & 0.9300 \\
\hline$N(53 A)-O(52 A)$ & $1.169(13)$ \\
\hline$N(53 A)-O(50 A)$ & $1.201(14)$ \\
\hline$N(53 A)-O(51 A)$ & $1.273(14)$ \\
\hline$N(53 B)-O(50 B)$ & $1.202(15)$ \\
\hline$N(53 B)-O(52 B)$ & $1.170(15)$ \\
\hline$N(53 B)-O(51 B)$ & $1.273(16)$ \\
\hline$C(52)-C(53)$ & $1.365(10)$ \\
\hline $\mathrm{C}(52)-\mathrm{H}(52)$ & 0.9300 \\
\hline$C(53)-C(54)$ & $1.390(13)$ \\
\hline$C(53)-C(57)$ & $1.518(11)$ \\
\hline$C(54)-C(55)$ & $1.364(12)$ \\
\hline $\mathrm{C}(54)-\mathrm{H}(54)$ & 0.9300 \\
\hline$C(55)-N(56)$ & $1.360(7)$ \\
\hline $\mathrm{C}(55)-\mathrm{H}(55)$ & 0.9300 \\
\hline$C(57)-H(57 A)$ & 0.9600 \\
\hline $\mathrm{C}(57)-\mathrm{H}(57 \mathrm{~B})$ & 0.9600 \\
\hline $\mathrm{C}(57)-\mathrm{H}(57 \mathrm{C})$ & 0.9600 \\
\hline$C(61 A)-N(66 A)$ & $1.315(15)$ \\
\hline$C(61 A)-C(62 A)$ & $1.398(14)$ \\
\hline$C(62 A)-C(63 A)$ & $1.380(10)$ \\
\hline$C(62 A)-H(62 A)$ & 0.9300 \\
\hline$C(63 A)-C(64 A)$ & $1.371(10)$ \\
\hline$C(63 A)-C(67 A)$ & $1.476(11)$ \\
\hline$C(64 A)-C(65 A)$ & $1.420(9)$ \\
\hline$C(64 A)-H(64 A)$ & 0.9300 \\
\hline$C(65 A)-N(66 A)$ & $1.335(10)$ \\
\hline$C(65 A)-H(65 A)$ & 0.9300 \\
\hline$C(67 A)-H(67 A)$ & 0.9600 \\
\hline$C(67 A)-H(67 B)$ & 0.9600 \\
\hline$C(67 A)-H(67 C)$ & 0.9600 \\
\hline$C(61 B)-N(66 B)$ & $1.326(16)$ \\
\hline$C(61 B)-C(62 B)$ & $1.393(14)$ \\
\hline$C(62 B)-C(63 B)$ & $1.381(10)$ \\
\hline$C(62 B)-H(62 B)$ & 0.9300 \\
\hline$C(63 B)-C(64 B)$ & $1.372(10)$ \\
\hline$C(63 B)-C(67 B)$ & $1.475(12)$ \\
\hline$C(64 B)-C(65 B)$ & $1.419(10)$ \\
\hline$C(64 B)-H(64 B)$ & 0.9300 \\
\hline$C(65 B)-N(66 B)$ & $1.332(11)$ \\
\hline$C(65 B)-H(65 B)$ & 0.9300 \\
\hline$C(67 B)-H(67 D)$ & 0.9600 \\
\hline$C(67 B)-H(67 E)$ & 0.9600 \\
\hline $\mathrm{C}(67 \mathrm{~B})-\mathrm{H}(67 \mathrm{~F})$ & 0.9600 \\
\hline $\mathrm{C}(45)-\mathrm{H}(45)$ & 0.9300 \\
\hline $\mathrm{N}(46) \# 2-\mathrm{Ag}(1)-\mathrm{N}(1)$ & $129.35(13)$ \\
\hline $\mathrm{N}(46) \# 2-\mathrm{Ag}(1)-\mathrm{N}(36) \# 2$ & $120.39(12)$ \\
\hline $\mathrm{N}(1)-\mathrm{Ag}(1)-\mathrm{N}(36) \# 2$ & $110.20(12)$ \\
\hline $\mathrm{N}(46) \# 2-\mathrm{Ag}(1)-\mathrm{N}(16)$ & $97.56(13)$ \\
\hline$N(1)-\operatorname{Ag}(1)-N(16)$ & $82.31(12)$ \\
\hline $\mathrm{N}(36) \# 2-\mathrm{Ag}(1)-\mathrm{N}(16)$ & $91.95(13)$ \\
\hline$P(1)-N(3)-P(3)$ & $123.1(2)$ \\
\hline$P(2)-N(2)-P(3)$ & $120.7(2)$ \\
\hline
\end{tabular}




\begin{tabular}{|c|c|}
\hline$P(2)-N(1)-P(1)$ & $123.2(2)$ \\
\hline $\mathrm{P}(2)-\mathrm{N}(1)-\mathrm{Ag}(1)$ & $113.12(18)$ \\
\hline$P(1)-N(1)-A g(1)$ & $122.23(19)$ \\
\hline$O(6)-P(3)-N(3)$ & $109.91(18)$ \\
\hline$O(6)-P(3)-N(2)$ & $109.52(18)$ \\
\hline$N(3)-P(3)-N(2)$ & $117.97(18)$ \\
\hline$O(6)-P(3)-O(5)$ & $94.99(16)$ \\
\hline$N(3)-P(3)-O(5)$ & $110.39(18)$ \\
\hline$N(2)-P(3)-O(5)$ & $111.65(17)$ \\
\hline$N(1)-P(2)-N(2)$ & $116.90(18)$ \\
\hline$N(1)-P(2)-O(4)$ & $108.25(17)$ \\
\hline $\mathrm{N}(2)-\mathrm{P}(2)-\mathrm{O}(4)$ & $110.49(17)$ \\
\hline$N(1)-P(2)-O(3)$ & $106.10(17)$ \\
\hline$N(2)-P(2)-O(3)$ & $114.36(18)$ \\
\hline$O(4)-P(2)-O(3)$ & $99.14(16)$ \\
\hline $\mathrm{N}(3)-\mathrm{P}(1)-\mathrm{N}(1)$ & $116.22(19)$ \\
\hline $\mathrm{N}(3)-\mathrm{P}(1)-\mathrm{O}(1)$ & $107.15(18)$ \\
\hline $\mathrm{N}(1)-\mathrm{P}(1)-\mathrm{O}(1)$ & $110.68(18)$ \\
\hline $\mathrm{N}(3)-\mathrm{P}(1)-\mathrm{O}(2)$ & $111.81(18)$ \\
\hline$N(1)-P(1)-O(2)$ & $104.91(18)$ \\
\hline $\mathrm{O}(1)-\mathrm{P}(1)-\mathrm{O}(2)$ & $105.62(16)$ \\
\hline $\mathrm{C}(31)-\mathrm{N}(36)-\mathrm{Ag}(1) \# 1$ & $134.8(3)$ \\
\hline $\mathrm{C}(35)-\mathrm{N}(36)-\mathrm{Ag}(1) \# 1$ & $108.7(3)$ \\
\hline $\mathrm{C}(41)-\mathrm{N}(46)-\mathrm{C}(45)$ & $116.9(4)$ \\
\hline $\mathrm{C}(41)-\mathrm{N}(46)-\mathrm{Ag}(1) \# 1$ & $111.6(3)$ \\
\hline $\mathrm{C}(45)-\mathrm{N}(46)-\mathrm{Ag}(1) \# 1$ & $131.5(3)$ \\
\hline $\mathrm{C}(31)-\mathrm{N}(36)-\mathrm{C}(35)$ & $115.8(4)$ \\
\hline$N(46)-C(45)-C(44)$ & $122.3(4)$ \\
\hline $\mathrm{N}(46)-\mathrm{C}(45)-\mathrm{H}(45)$ & 118.8 \\
\hline $\mathrm{C}(44)-\mathrm{C}(45)-\mathrm{H}(45)$ & 118.8 \\
\hline$N(36)-C(31)-C(32)$ & $125.4(4)$ \\
\hline $\mathrm{N}(36)-\mathrm{C}(31)-\mathrm{O}(3)$ & $119.5(4)$ \\
\hline$C(32)-C(31)-O(3)$ & $115.1(4)$ \\
\hline$C(33)-C(32)-C(31)$ & $118.2(4)$ \\
\hline $\mathrm{C}(33)-\mathrm{C}(32)-\mathrm{H}(32)$ & 120.9 \\
\hline $\mathrm{C}(31)-\mathrm{C}(32)-\mathrm{H}(32)$ & 120.9 \\
\hline$C(32)-C(33)-C(34)$ & $117.7(5)$ \\
\hline$C(32)-C(33)-C(37)$ & $121.7(5)$ \\
\hline$C(34)-C(33)-C(37)$ & $120.6(5)$ \\
\hline$C(35)-C(34)-C(33)$ & $119.5(5)$ \\
\hline $\mathrm{C}(35)-\mathrm{C}(34)-\mathrm{H}(34)$ & 120.3 \\
\hline $\mathrm{C}(33)-\mathrm{C}(34)-\mathrm{H}(34)$ & 120.3 \\
\hline$C(33)-C(37)-H(37 A)$ & 109.5 \\
\hline $\mathrm{C}(33)-\mathrm{C}(37)-\mathrm{H}(37 \mathrm{~B})$ & 109.5 \\
\hline $\mathrm{H}(37 \mathrm{~A})-\mathrm{C}(37)-\mathrm{H}(37 \mathrm{~B})$ & 109.5 \\
\hline $\mathrm{C}(33)-\mathrm{C}(37)-\mathrm{H}(37 \mathrm{C})$ & 109.5 \\
\hline $\mathrm{H}(37 \mathrm{~A})-\mathrm{C}(37)-\mathrm{H}(37 \mathrm{C})$ & 109.5 \\
\hline $\mathrm{H}(37 \mathrm{~B})-\mathrm{C}(37)-\mathrm{H}(37 \mathrm{C})$ & 109.5 \\
\hline$C(23)-C(22)-C(21)$ & $117.5(5)$ \\
\hline$C(23)-C(22)-H(22)$ & 121.2 \\
\hline $\mathrm{C}(21)-\mathrm{C}(22)-\mathrm{H}(22)$ & 121.2 \\
\hline$C(23)-C(27)-H(27 A)$ & 109.5 \\
\hline $\mathrm{C}(23)-\mathrm{C}(27)-\mathrm{H}(27 \mathrm{~B})$ & 109.5 \\
\hline $\mathrm{H}(27 \mathrm{~A})-\mathrm{C}(27)-\mathrm{H}(27 \mathrm{~B})$ & 109.5 \\
\hline $\mathrm{C}(23)-\mathrm{C}(27)-\mathrm{H}(27 \mathrm{C})$ & 109.5 \\
\hline $\mathrm{H}(27 \mathrm{~A})-\mathrm{C}(27)-\mathrm{H}(27 \mathrm{C})$ & 109.5 \\
\hline $\mathrm{H}(27 \mathrm{~B})-\mathrm{C}(27)-\mathrm{H}(27 \mathrm{C})$ & 109.5 \\
\hline $\mathrm{C}(24)-\mathrm{C}(25)-\mathrm{N}(26)$ & $123.4(5)$ \\
\hline $\mathrm{C}(24)-\mathrm{C}(25)-\mathrm{H}(25)$ & 118.3 \\
\hline $\mathrm{N}(26)-\mathrm{C}(25)-\mathrm{H}(25)$ & 118.3 \\
\hline$C(22)-C(23)-C(24)$ & $117.8(5)$ \\
\hline$C(22)-C(23)-C(27)$ & $120.8(6)$ \\
\hline$C(24)-C(23)-C(27)$ & $121.4(5)$ \\
\hline$C(25)-C(24)-C(23)$ & $119.6(5)$ \\
\hline $\mathrm{C}(25)-\mathrm{C}(24)-\mathrm{H}(24)$ & 120.2 \\
\hline $\mathrm{C}(23)-\mathrm{C}(24)-\mathrm{H}(24)$ & 120.2 \\
\hline$N(26)-C(21)-C(22)$ & $126.3(4)$ \\
\hline $\mathrm{N}(26)-\mathrm{C}(21)-\mathrm{O}(2)$ & $116.8(4)$ \\
\hline
\end{tabular}




\begin{tabular}{|c|c|}
\hline$C(22)-C(21)-O(2)$ & $116.8(4)$ \\
\hline$C(12)-C(13)-C(14)$ & $117.1(4)$ \\
\hline$C(12)-C(13)-C(17)$ & $122.1(5)$ \\
\hline$C(14)-C(13)-C(17)$ & $120.7(4)$ \\
\hline$C(11)-C(12)-C(13)$ & $118.5(4)$ \\
\hline $\mathrm{C}(11)-\mathrm{C}(12)-\mathrm{H}(12)$ & 120.8 \\
\hline $\mathrm{C}(13)-\mathrm{C}(12)-\mathrm{H}(12)$ & 120.8 \\
\hline$C(13)-C(17)-H(17 A)$ & 109.5 \\
\hline $\mathrm{C}(13)-\mathrm{C}(17)-\mathrm{H}(17 \mathrm{~B})$ & 109.5 \\
\hline $\mathrm{H}(17 \mathrm{~A})-\mathrm{C}(17)-\mathrm{H}(17 \mathrm{~B})$ & 109.5 \\
\hline $\mathrm{C}(13)-\mathrm{C}(17)-\mathrm{H}(17 \mathrm{C})$ & 109.5 \\
\hline $\mathrm{H}(17 \mathrm{~A})-\mathrm{C}(17)-\mathrm{H}(17 \mathrm{C})$ & 109.5 \\
\hline $\mathrm{H}(17 \mathrm{~B})-\mathrm{C}(17)-\mathrm{H}(17 \mathrm{C})$ & 109.5 \\
\hline$C(15)-C(14)-C(13)$ & $119.7(4)$ \\
\hline $\mathrm{C}(15)-\mathrm{C}(14)-\mathrm{H}(14)$ & 120.2 \\
\hline $\mathrm{C}(13)-\mathrm{C}(14)-\mathrm{H}(14)$ & 120.2 \\
\hline $\mathrm{N}(16)-\mathrm{C}(15)-\mathrm{C}(14)$ & $123.1(5)$ \\
\hline $\mathrm{N}(16)-\mathrm{C}(15)-\mathrm{H}(15)$ & 118.5 \\
\hline $\mathrm{C}(14)-\mathrm{C}(15)-\mathrm{H}(15)$ & 118.5 \\
\hline $\mathrm{N}(16)-\mathrm{C}(11)-\mathrm{C}(12)$ & $125.2(4)$ \\
\hline $\mathrm{N}(16)-\mathrm{C}(11)-\mathrm{O}(1)$ & $117.5(4)$ \\
\hline$C(12)-C(11)-O(1)$ & $117.2(4)$ \\
\hline$N(46)-C(41)-O(4)$ & $114.9(4)$ \\
\hline$N(46)-C(41)-C(42)$ & $124.6(4)$ \\
\hline$O(4)-C(41)-C(42)$ & $120.4(4)$ \\
\hline$N(56)-C(51)-C(52)$ & $125.5(5)$ \\
\hline$N(56)-C(51)-O(5)$ & $117.3(4)$ \\
\hline$C(52)-C(51)-O(5)$ & $117.2(5)$ \\
\hline$C(44)-C(43)-C(42)$ & $118.2(4)$ \\
\hline$C(44)-C(43)-C(47)$ & $122.2(5)$ \\
\hline$C(42)-C(43)-C(47)$ & $119.6(5)$ \\
\hline$C(43)-C(42)-C(41)$ & $117.8(4)$ \\
\hline $\mathrm{C}(43)-\mathrm{C}(42)-\mathrm{H}(42)$ & 121.1 \\
\hline $\mathrm{C}(41)-\mathrm{C}(42)-\mathrm{H}(42)$ & 121.1 \\
\hline$C(43)-C(47)-H(47 A)$ & 109.5 \\
\hline$C(43)-C(47)-H(47 B)$ & 109.5 \\
\hline $\mathrm{H}(47 \mathrm{~A})-\mathrm{C}(47)-\mathrm{H}(47 \mathrm{~B})$ & 109.5 \\
\hline$C(43)-C(47)-H(47 C)$ & 109.5 \\
\hline $\mathrm{H}(47 \mathrm{~A})-\mathrm{C}(47)-\mathrm{H}(47 \mathrm{C})$ & 109.5 \\
\hline $\mathrm{H}(47 \mathrm{~B})-\mathrm{C}(47)-\mathrm{H}(47 \mathrm{C})$ & 109.5 \\
\hline$N(36)-C(35)-C(34)$ & $123.4(4)$ \\
\hline $\mathrm{N}(36)-\mathrm{C}(35)-\mathrm{H}(35)$ & 118.3 \\
\hline $\mathrm{C}(34)-\mathrm{C}(35)-\mathrm{H}(35)$ & 118.3 \\
\hline$C(43)-C(44)-C(45)$ & $120.2(4)$ \\
\hline $\mathrm{C}(43)-\mathrm{C}(44)-\mathrm{H}(44)$ & 119.9 \\
\hline $\mathrm{C}(45)-\mathrm{C}(44)-\mathrm{H}(44)$ & 119.9 \\
\hline $\mathrm{C}(21)-\mathrm{N}(26)-\mathrm{C}(25)$ & $115.3(4)$ \\
\hline $\mathrm{C}(11)-\mathrm{N}(16)-\mathrm{C}(15)$ & $116.4(4)$ \\
\hline $\mathrm{C}(11)-\mathrm{N}(16)-\mathrm{Ag}(1)$ & $129.2(3)$ \\
\hline $\mathrm{C}(15)-\mathrm{N}(16)-\mathrm{Ag}(1)$ & $111.3(3)$ \\
\hline$C(21)-O(2)-P(1)$ & $121.2(3)$ \\
\hline$C(11)-O(1)-P(1)$ & $125.3(3)$ \\
\hline$C(61 B)-O(6)-C(61 A)$ & $10.8(10)$ \\
\hline$C(61 B)-O(6)-P(3)$ & $117.7(4)$ \\
\hline$C(61 A)-O(6)-P(3)$ & $124.8(2)$ \\
\hline$C(51)-O(5)-P(3)$ & $121.3(3)$ \\
\hline$C(31)-O(3)-P(2)$ & $127.5(3)$ \\
\hline$C(41)-O(4)-P(2)$ & $121.9(3)$ \\
\hline$O(52 A)-N(53 A)-O(50 A)$ & $130.2(15)$ \\
\hline$O(52 A)-N(53 A)-O(51 A)$ & $119.0(15)$ \\
\hline$O(50 A)-N(53 A)-O(51 A)$ & $110.8(13)$ \\
\hline$O(50 B)-N(53 B)-O(52 B)$ & $129.2(18)$ \\
\hline$O(50 B)-N(53 B)-O(51 B)$ & $112.1(15)$ \\
\hline$O(52 B)-N(53 B)-O(51 B)$ & $118.7(17)$ \\
\hline$C(53)-C(52)-C(51)$ & $118.1(7)$ \\
\hline $\mathrm{C}(53)-\mathrm{C}(52)-\mathrm{H}(52)$ & 120.9 \\
\hline $\mathrm{C}(51)-\mathrm{C}(52)-\mathrm{H}(52)$ & 120.9 \\
\hline$C(52)-C(53)-C(54)$ & $118.5(7)$ \\
\hline
\end{tabular}




$\begin{array}{ll}C(52)-C(53)-C(57) & 120.6(11) \\ C(54)-C(53)-C(57) & 120.9(9) \\ C(55)-C(54)-C(53) & 119.0(7) \\ C(55)-C(54)-H(54) & 120.5 \\ C(53)-C(54)-H(54) & 120.5 \\ N(56)-C(55)-C(54) & 123.6(8) \\ N(56)-C(55)-H(55) & 118.2 \\ C(54)-C(55)-H(55) & 118.2 \\ C(51)-N(56)-C(55) & 115.3(6) \\ C(53)-C(57)-H(57 A) & 109.5 \\ C(53)-C(57)-H(57 B) & 109.5 \\ H(57 A)-C(57)-H(57 B) & 109.5 \\ C(53)-C(57)-H(57 C) & 109.5 \\ H(57 A)-C(57)-H(57 C) & 109.5 \\ H(57 B)-C(57)-H(57 C) & 109.5 \\ N(66 A)-C(61 A)-C(62 A) & 126.5(6) \\ N(66 A)-C(61 A)-O(6) & 113.2(8) \\ C(62 A)-C(61 A)-O(6) & 120.3(10) \\ C(63 A)-C(62 A)-C(61 A) & 119.0(7) \\ C(63 A)-C(62 A)-H(62 A) & 120.5 \\ C(61 A)-C(62 A)-H(62 A) & 120.5 \\ C(64 A)-C(63 A)-C(62 A) & 115.6(8) \\ C(64 A)-C(63 A)-C(67 A) & 122.3(7) \\ C(62 A)-C(63 A)-C(67 A) & 122.1(7) \\ C(63 A)-C(64 A)-C(65 A) & 121.6(8) \\ C(63 A)-C(64 A)-H(64 A) & 119.2 \\ C(65 A)-C(64 A)-H(64 A) & 119.2 \\ N(66 A)-C(65 A)-C(64 A) & 122.4(7) \\ N(66 A)-C(65 A)-H(65 A) & 118.8 \\ C(64 A)-C(65 A)-H(65 A) & 118.8 \\ C(61 A)-N(66 A)-C(65 A) & 114.9(6) \\ N(66 B)-C(61 B)-C(62 B) & 126.3(7) \\ N(66 B)-C(61 B)-O(6) & 114.0(9) \\ C(62 B)-C(61 B)-O(6) & 119.7(10) \\ C(63 B)-C(62 B)-C(61 B) & 119.2(8) \\ C(63 B)-C(62 B)-H(62 B) & 120.4 \\ C(61 B)-C(62 B)-H(62 B) & 120.4 \\ C(64 B)-C(63 B)-C(62 B) & 115.5(8) \\ C(64 B)-C(63 B)-C(67 B) & 122.6(8) \\ C(62 B)-C(63 B)-C(67 B) & 121.9(7) \\ C(63 B)-C(64 B)-C(65 B) & 121.7(9) \\ C(63 B)-C(64 B)-H(64 B) & 119.2 \\ C(65 B)-C(64 B)-H(64 B) & 119.2 \\ N(66 B)-C(65 B)-C(64 B) & 122.5(8) \\ N(66 B)-C(65 B)-H(65 B) & 118.8 \\ C(64 B)-C(65 B)-H(65 B) & 118.8 \\ C(61 B)-N(66 B)-C(65 B) & 114.8(7) \\ & \end{array}$

Symmetry transformations used to generate equivalent atoms: $\# 1-x+3 / 2, y+1 / 2,-z+1 / 2, \# 2-x+3 / 2, y-1 / 2,-z+1 / 2$ 
Table 6. Anisotropic displacement parameters $\left(\AA^{2} \times 10^{3}\right)$ for $\left\{[\mathrm{Ag}(\mathrm{MeL})] \mathrm{NO}_{3}\right\}_{\infty} \cdot 1.33 \mathrm{CH}_{2} \mathrm{Cl}_{2}$ (3).

The anisotropic displacement factor exponent takes the form: $-2 \pi^{2}\left[h^{2} a{ }^{2} U^{11}+\ldots+2 h k a * b * U^{12}\right]$

\begin{tabular}{|c|c|c|c|c|c|c|}
\hline & $\mathrm{U}^{11}$ & $\mathrm{U}^{22}$ & $\mathrm{U}^{33}$ & $\mathrm{U}^{23}$ & $\mathrm{U}^{13}$ & $\mathrm{U}^{12}$ \\
\hline $\mathrm{N}(36)$ & $25(2)$ & $17(2)$ & $25(2)$ & $1(2)$ & $-2(2)$ & $-1(1)$ \\
\hline$N(46)$ & $22(2)$ & $20(2)$ & $28(2)$ & $1(2)$ & $-1(2)$ & $1(1)$ \\
\hline$C(45)$ & $28(2)$ & $25(2)$ & $40(3)$ & $4(2)$ & $-2(2)$ & $-6(2)$ \\
\hline $\operatorname{Ag}(1)$ & $34(1)$ & $14(1)$ & $29(1)$ & $3(1)$ & $-4(1)$ & $3(1)$ \\
\hline$C(31)$ & $27(2)$ & $18(2)$ & $22(2)$ & $4(2)$ & $-3(2)$ & $4(2)$ \\
\hline C (32) & $28(2)$ & $27(2)$ & $35(3)$ & $5(2)$ & $1(2)$ & $-2(2)$ \\
\hline C (33) & $38(3)$ & $38(3)$ & $32(3)$ & $3(2)$ & $7(2)$ & $1(2)$ \\
\hline C (34) & $46(3)$ & $36(3)$ & $29(3)$ & $-2(2)$ & $3(2)$ & $-3(2)$ \\
\hline C (37) & $50(4)$ & $63(4)$ & $47(3)$ & $5(3)$ & $12(3)$ & $-7(3)$ \\
\hline$C(22)$ & $41(3)$ & $30(3)$ & $27(3)$ & $-3(2)$ & $0(2)$ & $2(2)$ \\
\hline C (27) & $78(5)$ & $101(6)$ & $35(3)$ & $10(4)$ & $13(3)$ & $2(4)$ \\
\hline$C(25)$ & $41(3)$ & $29(3)$ & $34(3)$ & $1(2)$ & $-16(2)$ & $3(2)$ \\
\hline C (23) & $58(4)$ & $41(3)$ & $32(3)$ & $-3(2)$ & $5(3)$ & $-6(3)$ \\
\hline C (24) & $60(4)$ & $34(3)$ & $28(3)$ & $-1(2)$ & $-11(2)$ & $-2(3)$ \\
\hline C (21) & $41(3)$ & $14(2)$ & $25(2)$ & $-3(2)$ & $-7(2)$ & $0(2)$ \\
\hline$C(13)$ & $38(3)$ & $24(2)$ & $39(3)$ & $-2(2)$ & $-7(2)$ & $-8(2)$ \\
\hline$C(12)$ & $27(2)$ & $21(2)$ & $43(3)$ & $-1(2)$ & $-7(2)$ & $-2(2)$ \\
\hline C (17) & $44(3)$ & $28(3)$ & $83(4)$ & $-11(3)$ & $-23(3)$ & $-9(2)$ \\
\hline$C(14)$ & $47(3)$ & $14(2)$ & $44(3)$ & $0(2)$ & $-8(2)$ & $-6(2)$ \\
\hline C (15) & $54(3)$ & $11(2)$ & $53(3)$ & $2(2)$ & $-17(3)$ & $3(2)$ \\
\hline C (11) & $31(2)$ & $15(2)$ & $26(2)$ & $-1(2)$ & $-4(2)$ & $-6(2)$ \\
\hline C (41) & $21(2)$ & $18(2)$ & $23(2)$ & $1(2)$ & $-3(2)$ & $4(2)$ \\
\hline C (51) & $22(2)$ & $47(3)$ & $30(3)$ & $-3(2)$ & $-2(2)$ & $-5(2)$ \\
\hline$C(43)$ & $29(3)$ & $40(3)$ & $31(3)$ & $-3(2)$ & $1(2)$ & $7(2)$ \\
\hline$C(42)$ & $32(3)$ & $23(2)$ & $30(2)$ & $-7(2)$ & $-4(2)$ & $4(2)$ \\
\hline C (47) & $49(4)$ & $55(4)$ & $58(4)$ & $-18(3)$ & $11(3)$ & $11(3)$ \\
\hline C (35) & $42(3)$ & $23(2)$ & $25(2)$ & $1(2)$ & $-2(2)$ & $-4(2)$ \\
\hline C ( 44$)$ & $26(2)$ & $40(3)$ & $35(3)$ & $4(2)$ & $9(2)$ & $4(2)$ \\
\hline $\mathrm{N}(1)$ & $26(2)$ & $11(2)$ & $26(2)$ & $0(1)$ & $-5(2)$ & $-1(1)$ \\
\hline$N(26)$ & $37(2)$ & $23(2)$ & $29(2)$ & $-1(2)$ & $-7(2)$ & $3(2)$ \\
\hline$N(16)$ & $35(2)$ & $17(2)$ & $39(2)$ & $0(2)$ & $-14(2)$ & $0(2)$ \\
\hline $\mathrm{N}(3)$ & $29(2)$ & $10(2)$ & $30(2)$ & $3(1)$ & $-7(2)$ & $1(1)$ \\
\hline $\mathrm{N}(2)$ & $22(2)$ & $12(2)$ & $26(2)$ & $-2(1)$ & $-5(2)$ & $-1(1)$ \\
\hline$O(2)$ & $32(2)$ & $16(2)$ & $25(2)$ & $0(1)$ & $-4(1)$ & $3(1)$ \\
\hline$O(1)$ & $24(2)$ & $13(1)$ & $34(2)$ & $-4(1)$ & $-6(1)$ & $-1(1)$ \\
\hline$O(6)$ & $31(2)$ & $13(1)$ & $25(2)$ & $0(1)$ & $0(1)$ & $0(1)$ \\
\hline$O(5)$ & $22(2)$ & $19(2)$ & $31(2)$ & $-3(1)$ & $-2(1)$ & $3(1)$ \\
\hline$O(3)$ & $23(2)$ & $19(2)$ & $25(2)$ & $1(1)$ & $-3(1)$ & $-4(1)$ \\
\hline$O(4)$ & $21(2)$ & $16(1)$ & $23(2)$ & $0(1)$ & $-5(1)$ & $1(1)$ \\
\hline$N(53 A)$ & $35(7)$ & $56(5)$ & $52(4)$ & $-19(3)$ & $-8(4)$ & $-13(5)$ \\
\hline$O(52 A)$ & $80(7)$ & $149(11)$ & $174(8)$ & $-88(8)$ & $15(6)$ & $20(7)$ \\
\hline$O(50 A)$ & $73(6)$ & $105(8)$ & $89(7)$ & $-30(6)$ & $-6(5)$ & $10(5)$ \\
\hline$O(51 A)$ & $157(10)$ & $169(11)$ & $115(9)$ & $12(8)$ & $22(7)$ & $-9(8)$ \\
\hline$N(53 B)$ & $35(7)$ & $56(5)$ & $52(4)$ & $-19(3)$ & $-8(4)$ & $-13(5)$ \\
\hline$O(52 B)$ & $80(7)$ & $149(11)$ & $174(8)$ & $-88(8)$ & $15(6)$ & $20(7)$ \\
\hline$O(50 B)$ & $77(8)$ & $109(9)$ & $117(10)$ & $20(7)$ & $14(7)$ & $-32(7)$ \\
\hline$O(51 B)$ & $139(11)$ & $117(11)$ & $174(12)$ & $-22(8)$ & $-15(8)$ & $11(8)$ \\
\hline$P(3)$ & 21 (1) & $10(1)$ & $23(1)$ & $-1(1)$ & $-3(1)$ & $1(1)$ \\
\hline$P(2)$ & $19(1)$ & $12(1)$ & $23(1)$ & $0(1)$ & $-3(1)$ & $-1(1)$ \\
\hline P (1) & $22(1)$ & $11(1)$ & $25(1)$ & $-1(1)$ & $-6(1)$ & $0(1)$ \\
\hline$C(52)$ & $30(3)$ & $72(4)$ & $53(4)$ & $-34(3)$ & $-4(3)$ & $7(3)$ \\
\hline C (53) & $26(3)$ & $149(9)$ & $67(5)$ & $-60(5)$ & $7(3)$ & $-10(4)$ \\
\hline C (54) & $41(4)$ & $173(10)$ & $46(4)$ & $-11(5)$ & $12(3)$ & $-43(5)$ \\
\hline C (55) & $40(4)$ & $124(7)$ & $53(4)$ & $30(4)$ & $0(3)$ & $-35(4)$ \\
\hline$N(56)$ & $33(2)$ & $57(3)$ & $43(3)$ & $11(2)$ & $-5(2)$ & $-15(2)$ \\
\hline C (57) & $38(4)$ & $229(14)$ & $147(9)$ & $-115(9)$ & $21(5)$ & $13(6)$ \\
\hline$C(61 A)$ & $47(2)$ & $47(2)$ & $47(2)$ & $0(1)$ & $1(1)$ & $-1(1)$ \\
\hline$C(62 A)$ & $33(4)$ & $38(4)$ & $24(4)$ & $7(3)$ & $7(3)$ & $9(3)$ \\
\hline
\end{tabular}




\begin{tabular}{|c|c|c|c|c|c|c|}
\hline$C(63 A)$ & $38(4)$ & $31(4)$ & $26(4)$ & $12(3)$ & $-5(3)$ & $4(3)$ \\
\hline$C(64 A)$ & 38 (5) & $40(5)$ & $45(5)$ & $5(4)$ & $15(4)$ & $19(4)$ \\
\hline$C(65 A)$ & $40(4)$ & $65(6)$ & $43(5)$ & $9(4)$ & $-1(4)$ & $31(4)$ \\
\hline$N(66 A)$ & $34(3)$ & $57(4)$ & $24(3)$ & $4(3)$ & $8(3)$ & $17(3)$ \\
\hline$C(67 A)$ & $54(5)$ & $89(7)$ & $57(5)$ & $26(5)$ & $20(4)$ & $34(5)$ \\
\hline$C(61 B)$ & $47(2)$ & $47(2)$ & $47(2)$ & $0(1)$ & 1 (1) & $-1(1)$ \\
\hline$C(62 B)$ & $33(4)$ & $38(4)$ & $24(4)$ & $7(3)$ & $7(3)$ & $9(3)$ \\
\hline$C(63 B)$ & $38(4)$ & $31(4)$ & $26(4)$ & 12 (3) & $-5(3)$ & $4(3)$ \\
\hline$C(64 B)$ & $38(5)$ & $40(5)$ & $45(5)$ & $5(4)$ & $15(4)$ & $19(4)$ \\
\hline$C(65 B)$ & $40(4)$ & $65(6)$ & $43(5)$ & $9(4)$ & $-1(4)$ & 31 (4) \\
\hline $\mathrm{N}(66 \mathrm{~B})$ & $34(3)$ & $57(4)$ & $24(3)$ & $4(3)$ & $8(3)$ & $17(3)$ \\
\hline$C(67 B)$ & $54(5)$ & $89(7)$ & $57(5)$ & $26(5)$ & $20(4)$ & $34(5)$ \\
\hline
\end{tabular}


Table 7. Atomic coordinates $\left(x 10^{4}\right)$ and equivalent isotropic displacement parameters $\left(\AA^{2} \times 10^{3}\right)$ for $\left[\mathrm{Cu}_{2}(\mathrm{MeL})_{2}(\mu-\mathrm{OH})(\boldsymbol{\mu}-4-\mathrm{MeOpy})\right]\left(\mathrm{PF}_{6}\right)_{2} \cdot 2 \mathrm{CH}_{3} \mathrm{CN} \quad(\mathbf{5})$.

$\mathrm{U}(\mathrm{eq})$ is defined as one third of the trace of the orthogonalized $\mathrm{U}^{i j}$ tensor.

\begin{tabular}{|c|c|c|c|c|}
\hline & $\mathrm{x}$ & $\mathrm{y}$ & z & $\mathrm{U}(\mathrm{eq})$ \\
\hline C (15) & $409(6)$ & 3967 (3) & $1825(7)$ & $34(2)$ \\
\hline C (13) & $1206(5)$ & $3949(2)$ & $1069(6)$ & $21(2)$ \\
\hline$C(14)$ & $460(5)$ & $3980(2)$ & $1007(6)$ & $27(2)$ \\
\hline$C(16)$ & $1072(6)$ & $3926(2)$ & $2614(7)$ & $33(2)$ \\
\hline$C(18)$ & $-373(6)$ & $4010(3)$ & $1836(7)$ & $44(3)$ \\
\hline$C(17)$ & $1773(5)$ & $3903(2)$ & $2560(6)$ & $29(2)$ \\
\hline C (19) & $1280(5)$ & $3691(3)$ & $-1578(6)$ & $26(2)$ \\
\hline$N(12)$ & $1199(5)$ & $3824(2)$ & $-2370(6)$ & $39(2)$ \\
\hline$C(22)$ & $451(6)$ & $3227(3)$ & $-2966(7)$ & $40(3)$ \\
\hline$C(21)$ & $553(6)$ & $3093(3)$ & $-2144(7)$ & $36(3)$ \\
\hline C (23) & $777(7)$ & $3585(3)$ & $-3066(7)$ & $53(3)$ \\
\hline C (35) & $3478(5)$ & $3754(3)$ & $-1225(6)$ & $31(2)$ \\
\hline$C(31)$ & $3869(5)$ & $3351(2)$ & $-14(6)$ & $22(2)$ \\
\hline$C(32)$ & $4308(5)$ & $3112(2)$ & $-309(6)$ & $23(2)$ \\
\hline$C(34)$ & $3883(5)$ & $3530(3)$ & $-1582(6)$ & $29(2)$ \\
\hline C (33) & $4313(6)$ & $3199(3)$ & $-1128(6)$ & $34(3)$ \\
\hline$C(36)$ & $4767(6)$ & $2951(3)$ & $-1491(7)$ & $51(3)$ \\
\hline$C(12)$ & $5258(6)$ & $4463(3)$ & $-1090(6)$ & $45(3)$ \\
\hline$C(9)$ & $5163(5)$ & $4339(2)$ & $-269(6)$ & $25(2)$ \\
\hline$C(10)$ & $5616(5)$ & $4036(2)$ & $300(6)$ & $28(2)$ \\
\hline C (11) & $5507(5)$ & $3927(2)$ & $1036(6)$ & $25(2)$ \\
\hline$C(7)$ & $4514(5)$ & $4371(2)$ & $691(5)$ & $15(2)$ \\
\hline$C(25)$ & $3124(5)$ & $2845(2)$ & $2039(6)$ & $23(2)$ \\
\hline$C(26)$ & $2973(5)$ & $2774(3)$ & $2764(6)$ & $25(2)$ \\
\hline C (27) & $2651(5)$ & $2403(3)$ & $2833(6)$ & $29(2)$ \\
\hline$C(30)$ & $2447(6)$ & $2319(3)$ & $3597(7)$ & $44(3)$ \\
\hline$C(28)$ & $2529(5)$ & $2139(3)$ & $2132(7)$ & $37(3)$ \\
\hline C (29) & $2701(6)$ & $2237(3)$ & $1435(7)$ & $44(3)$ \\
\hline$C(78)$ & $4164(5)$ & $3871(2)$ & $3663(5)$ & $20(2)$ \\
\hline$C(73)$ & $5049(5)$ & $3354(2)$ & $3884(5)$ & $20(2)$ \\
\hline$C(74)$ & $4797(5)$ & $3184(2)$ & $4500(6)$ & $23(2)$ \\
\hline C (77) & $3919(5)$ & $3723(2)$ & $4267(5)$ & $22(2)$ \\
\hline$C(75)$ & $4246(5)$ & $3352(2)$ & $4704(6)$ & $21(2)$ \\
\hline$C(76)$ & $3955(5)$ & $3168(2)$ & $5342(6)$ & $33(2)$ \\
\hline C (47) & $6264(6)$ & $4116(2)$ & $5203(6)$ & $27(2)$ \\
\hline$C(46)$ & $6207(6)$ & $4501(2)$ & $5473(6)$ & $28(2)$ \\
\hline C (45) & $6814(6)$ & $4774(2)$ & $5664(6)$ & $30(2)$ \\
\hline$C(48)$ & $6788(6)$ & $5192(2)$ & $5988(7)$ & $43(3)$ \\
\hline C ( 44$)$ & $7437(6)$ & $4638(2)$ & $5546(6)$ & $31(2)$ \\
\hline C (43) & $7440(6)$ & $4242(3)$ & $5268(6)$ & $29(2)$ \\
\hline$C(67)$ & $9625(6)$ & $4433(3)$ & $4034(6)$ & $29(2)$ \\
\hline C (68) & $10061(5)$ & $4695(3)$ & $3792(6)$ & $32(2)$ \\
\hline C (69) & $9822(6)$ & $5094(2)$ & $3647(6)$ & $28(2)$ \\
\hline$C(70)$ & $9162(6)$ & $5189(3)$ & $3764(6)$ & $33(3)$ \\
\hline C (71) & $8777(5)$ & $4904(3)$ & $3998(6)$ & $28(2)$ \\
\hline$C(72)$ & $10250(6)$ & $5402(3)$ & $3368(6)$ & $41(3)$ \\
\hline$C(6)$ & $4836(6)$ & $5878(2)$ & $3327(6)$ & $30(2)$ \\
\hline C (61) & $9834(5)$ & $2937(3)$ & $4565(6)$ & $25(2)$ \\
\hline C (62) & $10473(5)$ & $2728(3)$ & $5147(6)$ & $30(2)$ \\
\hline C (63) & $10354(6)$ & $2327(2)$ & $5349(6)$ & $31(2)$ \\
\hline$C(66)$ & $11022(6)$ & $2089(3)$ & $5997(7)$ & $46(3)$ \\
\hline C (64) & $9625(6)$ & $2176(3)$ & $4927(7)$ & $33(3)$ \\
\hline C (65) & $9032(6)$ & $2417(3)$ & $4372(6)$ & $30(2)$ \\
\hline C (37) & $7792(5)$ & $3012(2)$ & $5277(6)$ & $20(2)$ \\
\hline C (38) & $8246(5)$ & $2706(2)$ & $5823(6)$ & $27(2)$ \\
\hline C (39) & $7950(5)$ & $2317(2)$ & $5641(6)$ & $24(2)$ \\
\hline C (42) & $8419(5)$ & $1975(2)$ & $6192(6)$ & $34(3)$ \\
\hline
\end{tabular}




\begin{tabular}{|c|c|c|c|c|}
\hline$C(40)$ & $7229(5)$ & $2270(3)$ & $4971(6)$ & $27(2)$ \\
\hline$C(41)$ & $6816(5)$ & $2591(2)$ & $4465(6)$ & $28(2)$ \\
\hline$C(49)$ & $6986(5)$ & $2860(2)$ & $2398(6)$ & $21(2)$ \\
\hline$C(50)$ & 7077 (5) & $2465(2)$ & 2155 (5) & $23(2)$ \\
\hline$C(51)$ & $6439(5)$ & $2213(2)$ & $1909(6)$ & $22(2)$ \\
\hline$C(54)$ & $6492(6)$ & $1781(2)$ & $1646(6)$ & $39(3)$ \\
\hline$C(52)$ & $5762(6)$ & $2376(3)$ & $1901(6)$ & $33(2)$ \\
\hline$C(53)$ & $5757(5)$ & 2771 (3) & $2171(6)$ & $26(2)$ \\
\hline$C(4)$ & $5484(5)$ & $5201(2)$ & $3493(6)$ & $20(2)$ \\
\hline$C(55)$ & $7296(5)$ & $4216(2)$ & $2133(6)$ & $21(2)$ \\
\hline$C(1)$ & $4143(5)$ & $4831(2)$ & $2488(5)$ & $20(2)$ \\
\hline$C(2)$ & $4119(5)$ & $5240(2)$ & $2639(6)$ & $21(2)$ \\
\hline$C(59)$ & $6923(5)$ & $4822(2)$ & $2410(6)$ & $29(2)$ \\
\hline$C(56)$ & $7718(5)$ & $4376(2)$ & $1715(6)$ & $26(2)$ \\
\hline$C(57)$ & $7747(5)$ & $4789(2)$ & $1645(6)$ & $27(2)$ \\
\hline$C(8)$ & $4593(5)$ & $4503(2)$ & $-58(6)$ & $24(2)$ \\
\hline$C(3)$ & $4811(5)$ & $5430(2)$ & $3165(6)$ & $24(2)$ \\
\hline$C(5)$ & $5433(5)$ & $4793(2)$ & $3289(6)$ & $22(2)$ \\
\hline$C(60)$ & $8212(6)$ & $4981(3)$ & $1211(7)$ & $48(3)$ \\
\hline$C(58)$ & $7329(6)$ & $5008(3)$ & $2013(6)$ & $33(3)$ \\
\hline$C(24)$ & $257(6)$ & $2694(3)$ & $-2008(7)$ & $50(3)$ \\
\hline$C(90)$ & $8768(7)$ & $3534(4)$ & $7757(8)$ & $56(3)$ \\
\hline$C(91)$ & $8962(9)$ & $3944(3)$ & 7864 (11) & $108(6)$ \\
\hline $\mathrm{Cu}(1)$ & $4892(1)$ & 3935 (1) & $2438(1)$ & $19(1)$ \\
\hline $\mathrm{Cu}(2)$ & $6521(1)$ & $3438(1)$ & $3781(1)$ & $20(1)$ \\
\hline$F(1)$ & $7147(4)$ & $653(2)$ & $4262(4)$ & $67(2)$ \\
\hline $\mathrm{F}(2)$ & $7368(4)$ & $958(2)$ & $3171(4)$ & $62(2)$ \\
\hline F (3) & $8267(4)$ & $977(2)$ & $4604(4)$ & $76(2)$ \\
\hline F (4) & $7377(4)$ & $1235(2)$ & $5031(4)$ & $62(2)$ \\
\hline F (5) & $7628(4)$ & $1540(2)$ & 3955 (4) & $56(2)$ \\
\hline$F(6)$ & $6506(4)$ & $1222(2)$ & $3616(4)$ & $66(2)$ \\
\hline$F(7 A)$ & $2524(7)$ & $820(3)$ & $1698(6)$ & $79(5)$ \\
\hline$F(8 A)$ & $2672(5)$ & $457(2)$ & $656(7)$ & $54(3)$ \\
\hline$F(9 A)$ & $3353(5)$ & $1020(3)$ & $1151(8)$ & $80(5)$ \\
\hline $\mathrm{F}(10 \mathrm{~A})$ & $2411(6)$ & $1003(3)$ & $-229(6)$ & $72(4)$ \\
\hline$F(11 A)$ & $2276(7)$ & $1370(2)$ & $855(8)$ & $81(5)$ \\
\hline$F(12 A)$ & $1585(5)$ & $806(3)$ & $326(7)$ & $61(4)$ \\
\hline$F(7 B)$ & $2696(9)$ & $637(4)$ & $1415(10)$ & $63(7)$ \\
\hline$F(8 B)$ & $1868(9)$ & $669(4)$ & $-48(10)$ & $84(7)$ \\
\hline$F(9 B)$ & $3111(8)$ & $836(4)$ & $398(10)$ & $47(5)$ \\
\hline $\mathrm{F}(10 \mathrm{~B})$ & $2216(8)$ & $1308(4)$ & $-90(8)$ & $52(5)$ \\
\hline$F(11 B)$ & $3026(10)$ & $1285(4)$ & $1341(9)$ & $67(6)$ \\
\hline $\mathrm{F}(12 \mathrm{~B})$ & $1795(10)$ & $1118(6)$ & $930(12)$ & $106(9)$ \\
\hline $\mathrm{N}(1)$ & $3742(4)$ & $3900(2)$ & $1626(4)$ & $16(2)$ \\
\hline$N(3)$ & $2459(4)$ & $3508(2)$ & $509(5)$ & $23(2)$ \\
\hline$N(2)$ & $2522(4)$ & $4331(2)$ & $516(5)$ & $22(2)$ \\
\hline$N(4)$ & $7580(4)$ & $3603(2)$ & $3841(4)$ & $18(2)$ \\
\hline$N(6)$ & $9085(4)$ & $3676(2)$ & $4973(4)$ & $22(2)$ \\
\hline N ( 5) & $8664(4)$ & $3645(2)$ & $3196(5)$ & $21(2)$ \\
\hline $\mathrm{N}(17)$ & $9130(4)$ & $2806(2)$ & 4191 (5) & $28(2)$ \\
\hline$N(18)$ & $9004(4)$ & $4511(2)$ & $4135(5)$ & $28(2)$ \\
\hline$N(16)$ & $6892(4)$ & $4418(2)$ & $2478(5)$ & $24(2)$ \\
\hline$N(14)$ & $6860(4)$ & $3982(2)$ & $5081(4)$ & $23(2)$ \\
\hline$N(13)$ & $7102(4)$ & $2977(2)$ & $4622(5)$ & $21(2)$ \\
\hline$N(19)$ & $4698(4)$ & $3706(2)$ & $3453(5)$ & $20(2)$ \\
\hline$N(7)$ & $4778(4)$ & $4606(2)$ & $2800(4)$ & $20(2)$ \\
\hline $\mathrm{N}(8)$ & $4972(4)$ & $4100(2)$ & $1271(5)$ & $18(2)$ \\
\hline$N(10)$ & $3474(4)$ & $3676(2)$ & $-425(5)$ & $26(2)$ \\
\hline $\mathrm{N}(11)$ & $1855(4)$ & $3914(2)$ & $1789(5)$ & $26(2)$ \\
\hline$N(9)$ & $3010(4)$ & $2596(2)$ & $1367(5)$ & $31(2)$ \\
\hline$C(20)$ & $974(6)$ & $3344(3)$ & $-1414(7)$ & $37(3)$ \\
\hline$N(15)$ & $6375(4)$ & $3022(2)$ & $2427(5)$ & $25(2)$ \\
\hline$N(90)$ & $8595(6)$ & $3190(3)$ & $7650(6)$ & $65(3)$ \\
\hline$O(14)$ & $5974(3)$ & $3886(1)$ & $3080(3)$ & $18(1)$ \\
\hline$O(13)$ & $5574(3)$ & $3190(2)$ & $3701(4)$ & $27(2)$ \\
\hline$O(9)$ & $7641(3)$ & $3099(1)$ & $2625(4)$ & $23(2)$ \\
\hline$O(10)$ & $7237(3)$ & $3788(1)$ & $2175(4)$ & $20(1)$ \\
\hline$O(12)$ & $9891(3)$ & $4032(2)$ & $4194(4)$ & $27(2)$ \\
\hline
\end{tabular}




\begin{tabular}{lrrrr}
$O(11)$ & $9972(3)$ & $3335(2)$ & $4369(4)$ & $28(2)$ \\
$O(7)$ & $8089(3)$ & $3405(2)$ & $5500(4)$ & $25(2)$ \\
$O(8)$ & $8100(3)$ & $4119(2)$ & $5182(4)$ & $24(2)$ \\
$O(1)$ & $3449(3)$ & $4638(1)$ & $1976(4)$ & $20(1)$ \\
$O(3)$ & $1246(3)$ & $3948(2)$ & $238(4)$ & $25(1)$ \\
$O(4)$ & $1729(3)$ & $3963(2)$ & $-902(4)$ & $25(2)$ \\
$O(6)$ & $3848(3)$ & $3249(2)$ & $793(4)$ & $22(1)$ \\
$O(5)$ & $3465(3)$ & $3217(2)$ & $2020(4)$ & $25(2)$ \\
$O(2)$ & $3930(3)$ & $4549(1)$ & $858(4)$ & $20(1)$ \\
$P(5)$ & $7813(1)$ & $3544(1)$ & $3006(2)$ & $20(1)$ \\
$P(6)$ & $9334(1)$ & $3674(1)$ & $4169(2)$ & $24(1)$ \\
$P(4)$ & $8226(1)$ & $3696(1)$ & $4819(2)$ & $22(1)$ \\
$P(1)$ & $3373(1)$ & $4326(1)$ & $1211(2)$ & $21(1)$ \\
$P(2)$ & $2039(1)$ & $3928(1)$ & $141(2)$ & $24(1)$ \\
$P(3)$ & $3319(1)$ & $3483(1)$ & $1183(2)$ & $21(1)$ \\
$P(7)$ & $7382(2)$ & $1097(1)$ & $4108(2)$ & $46(1)$ \\
$P(8)$ & $2456(2)$ & $941(1)$ & $711(2)$ & $38(1)$ \\
$C(95 A)$ & $8703(12)$ & $4981(5)$ & $9176(13)$ & $114(6)$ \\
$N(95 A)$ & $8640(30)$ & $4998(14)$ & $7900(30)$ & $168(10)$ \\
$C(96 A)$ & $8510(30)$ & $4958(14)$ & $8460(40)$ & $113(8)$ \\
$C(95 B)$ & $8703(12)$ & $4981(5)$ & $9176(13)$ & $114(6)$ \\
$N(95 B)$ & $9820(18)$ & $4859(8)$ & $8839(18)$ & $168(10)$ \\
$C(96 B)$ & $9340(20)$ & $4921(8)$ & $9014(19)$ & $113(8)$ \\
\hline
\end{tabular}


Table 8. Bond lengths $[\AA]$ and angles $\left[{ }^{\circ}\right]$ for $\left[\mathrm{Cu}_{2}(\mathrm{MeL})_{2}(\boldsymbol{\mu}-\mathrm{OH})(\boldsymbol{\mu}-4-\mathrm{MeOpy})\right]\left(\mathrm{PF}_{6}\right)_{2} \cdot 2 \mathrm{CH}_{3} \mathrm{CN} \quad(\mathbf{5})$.

\begin{tabular}{|c|c|}
\hline $\mathrm{Cu}(1)-\mathrm{O}(14)$ & $1.900(5)$ \\
\hline $\mathrm{Cu}(1)-\mathrm{N}(19)$ & $2.014(7)$ \\
\hline $\mathrm{Cu}(1)-\mathrm{N}(1)$ & $2.046(7)$ \\
\hline $\mathrm{Cu}(1)-\mathrm{N}(8)$ & $2.069(7)$ \\
\hline $\mathrm{Cu}(1)-\mathrm{N}(7)$ & $2.343(6)$ \\
\hline $\mathrm{Cu}(2)-\mathrm{O}(14)$ & $1.908(5)$ \\
\hline $\mathrm{Cu}(2)-\mathrm{O}(13)$ & $1.948(6)$ \\
\hline $\mathrm{Cu}(2)-\mathrm{N}(13)$ & $2.052(7)$ \\
\hline $\mathrm{Cu}(2)-\mathrm{N}(4)$ & $2.067(7)$ \\
\hline $\mathrm{N}(1)-\mathrm{P}(1)$ & $1.603(6)$ \\
\hline$N(1)-P(3)$ & $1.619(6)$ \\
\hline $\mathrm{N}(3)-\mathrm{P}(3)$ & $1.556(7)$ \\
\hline$N(3)-P(2)$ & $1.599(6)$ \\
\hline $\mathrm{N}(2)-\mathrm{P}(1)$ & $1.550(7)$ \\
\hline $\mathrm{N}(2)-\mathrm{P}(2)$ & $1.599(7)$ \\
\hline $\mathrm{N}(4)-\mathrm{P}(4)$ & $1.597(7)$ \\
\hline $\mathrm{N}(4)-\mathrm{P}(5)$ & $1.630(7)$ \\
\hline $\mathrm{N}(6)-\mathrm{P}(4)$ & $1.561(7)$ \\
\hline $\mathrm{N}(6)-\mathrm{P}(6)$ & $1.588(7)$ \\
\hline$N(5)-P(5)$ & $1.564(7)$ \\
\hline $\mathrm{N}(5)-\mathrm{P}(6)$ & $1.582(7)$ \\
\hline $\mathrm{O}(9)-\mathrm{P}(5)$ & $1.588(5)$ \\
\hline $\mathrm{O}(10)-\mathrm{P}(5)$ & $1.576(6)$ \\
\hline $\mathrm{O}(12)-\mathrm{P}(6)$ & $1.590(5)$ \\
\hline $\mathrm{O}(11)-\mathrm{P}(6)$ & $1.593(6)$ \\
\hline $\mathrm{O}(7)-\mathrm{P}(4)$ & $1.587(5)$ \\
\hline $\mathrm{O}(8)-\mathrm{P}(4)$ & $1.586(5)$ \\
\hline $\mathrm{O}(1)-\mathrm{P}(1)$ & $1.592(5)$ \\
\hline $\mathrm{O}(3)-\mathrm{P}(2)$ & $1.598(6)$ \\
\hline $\mathrm{O}(4)-\mathrm{P}(2)$ & $1.570(6)$ \\
\hline $\mathrm{O}(6)-\mathrm{P}(3)$ & $1.613(6)$ \\
\hline$O(5)-P(3)$ & $1.562(6)$ \\
\hline$O(2)-P(1)$ & $1.601(6)$ \\
\hline$C(15)-C(16)$ & $1.391(13)$ \\
\hline$C(15)-C(14)$ & $1.396(12)$ \\
\hline$C(15)-C(18)$ & $1.515(12)$ \\
\hline $\mathrm{C}(13)-\mathrm{N}(11)$ & $1.315(10)$ \\
\hline$C(13)-C(14)$ & $1.395(11)$ \\
\hline$C(13)-O(3)$ & $1.406(9)$ \\
\hline$C(16)-C(17)$ & $1.388(12)$ \\
\hline $\mathrm{C}(17)-\mathrm{N}(11)$ & $1.348(10)$ \\
\hline $\mathrm{C}(19)-\mathrm{N}(12)$ & $1.326(10)$ \\
\hline$C(19)-C(20)$ & $1.374(12)$ \\
\hline $\mathrm{C}(19)-\mathrm{O}(4)$ & $1.411(10)$ \\
\hline $\mathrm{N}(12)-\mathrm{C}(23)$ & $1.350(11)$ \\
\hline$C(22)-C(21)$ & $1.361(12)$ \\
\hline$C(22)-C(23)$ & $1.388(13)$ \\
\hline$C(21)-C(20)$ & $1.406(12)$ \\
\hline $\mathrm{C}(21)-\mathrm{C}(24)$ & $1.499(12)$ \\
\hline $\mathrm{C}(35)-\mathrm{N}(10)$ & $1.349(10)$ \\
\hline$C(35)-C(34)$ & $1.377(12)$ \\
\hline $\mathrm{C}(31)-\mathrm{N}(10)$ & $1.329(10)$ \\
\hline$C(31)-C(32)$ & $1.389(11)$ \\
\hline$C(31)-O(6)$ & $1.392(9)$ \\
\hline$C(32)-C(33)$ & $1.386(12)$ \\
\hline$C(34)-C(33)$ & $1.390(12)$ \\
\hline$C(33)-C(36)$ & $1.497(12)$ \\
\hline$C(12)-C(9)$ & $1.499(11)$ \\
\hline$C(9)-C(8)$ & $1.392(11)$ \\
\hline$C(9)-C(10)$ & $1.400(12)$ \\
\hline$C(10)-C(11)$ & $1.366(11)$ \\
\hline $\mathrm{C}(11)-\mathrm{N}(8)$ & $1.369(10)$ \\
\hline $\mathrm{C}(7)-\mathrm{N}(8)$ & $1.338(10)$ \\
\hline
\end{tabular}




\begin{tabular}{|c|c|}
\hline$C(7)-C(8)$ & $1.379(10)$ \\
\hline$C(7)-O(2)$ & $1.395(9)$ \\
\hline $\mathrm{C}(25)-\mathrm{N}(9)$ & $1.327(10)$ \\
\hline$C(25)-C(26)$ & $1.365(11)$ \\
\hline$C(25)-O(5)$ & $1.407(9)$ \\
\hline$C(26)-C(27)$ & $1.406(11)$ \\
\hline$C(27)-C(28)$ & $1.390(12)$ \\
\hline$C(27)-C(30)$ & $1.496(12)$ \\
\hline$C(28)-C(29)$ & $1.364(13)$ \\
\hline $\mathrm{C}(29)-\mathrm{N}(9)$ & $1.357(11)$ \\
\hline $\mathrm{C}(78)-\mathrm{N}(19)$ & $1.332(10)$ \\
\hline$C(78)-C(77)$ & $1.360(10)$ \\
\hline$C(73)-O(13)$ & $1.290(9)$ \\
\hline $\mathrm{C}(73)-\mathrm{N}(19)$ & $1.387(10)$ \\
\hline$C(73)-C(74)$ & $1.413(11)$ \\
\hline$C(74)-C(75)$ & $1.358(11)$ \\
\hline$C(77)-C(75)$ & $1.433(11)$ \\
\hline$C(75)-C(76)$ & $1.512(11)$ \\
\hline $\mathrm{C}(47)-\mathrm{N}(14)$ & $1.322(10)$ \\
\hline$C(47)-C(46)$ & $1.375(11)$ \\
\hline$C(46)-C(45)$ & $1.403(12)$ \\
\hline$C(45)-C(44)$ & $1.367(12)$ \\
\hline$C(45)-C(48)$ & $1.498(11)$ \\
\hline$C(44)-C(43)$ & $1.396(11)$ \\
\hline $\mathrm{C}(43)-\mathrm{N}(14)$ & $1.338(10)$ \\
\hline$C(43)-O(8)$ & $1.393(10)$ \\
\hline $\mathrm{C}(67)-\mathrm{N}(18)$ & $1.299(11)$ \\
\hline$C(67)-C(68)$ & $1.377(12)$ \\
\hline$C(67)-O(12)$ & $1.415(9)$ \\
\hline$C(68)-C(69)$ & $1.394(11)$ \\
\hline$C(69)-C(70)$ & $1.396(12)$ \\
\hline$C(69)-C(72)$ & $1.500(11)$ \\
\hline$C(70)-C(71)$ & $1.356(12)$ \\
\hline $\mathrm{C}(71)-\mathrm{N}(18)$ & $1.365(10)$ \\
\hline$C(6)-C(3)$ & $1.511(10)$ \\
\hline $\mathrm{C}(61)-\mathrm{N}(17)$ & $1.303(11)$ \\
\hline$C(61)-C(62)$ & $1.384(12)$ \\
\hline$C(61)-O(11)$ & $1.412(9)$ \\
\hline$C(62)-C(63)$ & $1.416(11)$ \\
\hline$C(63)-C(64)$ & $1.369(12)$ \\
\hline$C(63)-C(66)$ & $1.505(12)$ \\
\hline$C(64)-C(65)$ & $1.378(12)$ \\
\hline $\mathrm{C}(65)-\mathrm{N}(17)$ & $1.356(10)$ \\
\hline $\mathrm{C}(37)-\mathrm{N}(13)$ & $1.314(11)$ \\
\hline$C(37)-C(38)$ & $1.396(11)$ \\
\hline$C(37)-O(7)$ & $1.411(9)$ \\
\hline$C(38)-C(39)$ & $1.394(11)$ \\
\hline$C(39)-C(40)$ & $1.369(12)$ \\
\hline$C(39)-C(42)$ & $1.497(11)$ \\
\hline$C(40)-C(41)$ & $1.381(11)$ \\
\hline $\mathrm{C}(41)-\mathrm{N}(13)$ & $1.377(10)$ \\
\hline $\mathrm{C}(49)-\mathrm{N}(15)$ & $1.310(10)$ \\
\hline$C(49)-C(50)$ & $1.403(11)$ \\
\hline$C(49)-O(9)$ & $1.399(9)$ \\
\hline$C(50)-C(51)$ & $1.396(11)$ \\
\hline$C(51)-C(52)$ & $1.403(12)$ \\
\hline$C(51)-C(54)$ & $1.516(11)$ \\
\hline$C(52)-C(53)$ & $1.390(11)$ \\
\hline $\mathrm{C}(53)-\mathrm{N}(15)$ & $1.363(10)$ \\
\hline$C(4)-C(5)$ & $1.390(10)$ \\
\hline$C(4)-C(3)$ & $1.397(11)$ \\
\hline $\mathrm{C}(55)-\mathrm{N}(16)$ & $1.321(10)$ \\
\hline$C(55)-C(56)$ & $1.374(11)$ \\
\hline$C(55)-O(10)$ & $1.432(9)$ \\
\hline$C(1)-N(7)$ & $1.334(10)$ \\
\hline$C(1)-C(2)$ & $1.387(10)$ \\
\hline$C(1)-O(1)$ & $1.399(9)$ \\
\hline$C(2)-C(3)$ & $1.395(12)$ \\
\hline
\end{tabular}




\begin{tabular}{|c|c|}
\hline $\mathrm{C}(59)-\mathrm{N}(16)$ & $1.354(9)$ \\
\hline$C(59)-C(58)$ & $1.358(12)$ \\
\hline$C(56)-C(57)$ & $1.381(11)$ \\
\hline$C(57)-C(58)$ & $1.398(12)$ \\
\hline$C(57)-C(60)$ & $1.502(12)$ \\
\hline$C(5)-N(7)$ & $1.330(10)$ \\
\hline$C(90)-N(90)$ & $1.184(12)$ \\
\hline$C(90)-C(91)$ & $1.403(15)$ \\
\hline$F(1)-P(7)$ & $1.599(6)$ \\
\hline$F(2)-P(7)$ & $1.604(6)$ \\
\hline$F(3)-P(7)$ & $1.594(7)$ \\
\hline $\mathrm{F}(4)-\mathrm{P}(7)$ & $1.595(6)$ \\
\hline$F(5)-P(7)$ & $1.597(6)$ \\
\hline$F(6)-P(7)$ & $1.584(7)$ \\
\hline$F(7 A)-P(8)$ & $1.629(9)$ \\
\hline$F(8 A)-P(8)$ & $1.673(7)$ \\
\hline$F(9 A)-P(8)$ & $1.584(9)$ \\
\hline$F(10 A)-P(8)$ & $1.532(8)$ \\
\hline$F(11 A)-P(8)$ & $1.511(8)$ \\
\hline$F(12 A)-P(8)$ & $1.582(8)$ \\
\hline$F(7 B)-P(8)$ & $1.459(10)$ \\
\hline$F(8 B)-P(8)$ & $1.571(12)$ \\
\hline$F(9 B)-P(8)$ & $1.587(10)$ \\
\hline$F(10 B)-P(8)$ & $1.713(11)$ \\
\hline$F(11 B)-P(8)$ & $1.622(11)$ \\
\hline$F(12 B)-P(8)$ & $1.572(11)$ \\
\hline$C(95 A)-C(96 A)$ & $1.09(6)$ \\
\hline$N(95 A)-C(96 A)$ & $1.06(6)$ \\
\hline$N(95 B)-C(96 B)$ & $1.10(3)$ \\
\hline $\mathrm{O}(14)-\mathrm{Cu}(1)-\mathrm{N}(19)$ & $92.6(3)$ \\
\hline $\mathrm{O}(14)-\mathrm{Cu}(1)-\mathrm{N}(1)$ & $169.8(2)$ \\
\hline $\mathrm{N}(19)-\mathrm{Cu}(1)-\mathrm{N}(1)$ & $89.8(3)$ \\
\hline $\mathrm{O}(14)-\mathrm{Cu}(1)-\mathrm{N}(8)$ & $92.8(2)$ \\
\hline $\mathrm{N}(19)-\mathrm{Cu}(1)-\mathrm{N}(8)$ & $170.7(3)$ \\
\hline $\mathrm{N}(1)-\mathrm{Cu}(1)-\mathrm{N}(8)$ & $83.6(3)$ \\
\hline $\mathrm{O}(14)-\mathrm{Cu}(1)-\mathrm{N}(7)$ & $97.9(2)$ \\
\hline $\mathrm{N}(19)-\mathrm{Cu}(1)-\mathrm{N}(7)$ & $94.5(2)$ \\
\hline $\mathrm{N}(1)-\mathrm{Cu}(1)-\mathrm{N}(7)$ & $91.7(2)$ \\
\hline $\mathrm{N}(8)-\mathrm{Cu}(1)-\mathrm{N}(7)$ & $92.1(2)$ \\
\hline $\mathrm{O}(14)-\mathrm{Cu}(2)-\mathrm{O}(13)$ & $91.7(2)$ \\
\hline $\mathrm{O}(14)-\mathrm{Cu}(2)-\mathrm{N}(13)$ & $175.4(2)$ \\
\hline $\mathrm{O}(13)-\mathrm{Cu}(2)-\mathrm{N}(13)$ & $87.5(3)$ \\
\hline $\mathrm{O}(14)-\mathrm{Cu}(2)-\mathrm{N}(4)$ & $95.7(2)$ \\
\hline $\mathrm{O}(13)-\mathrm{Cu}(2)-\mathrm{N}(4)$ & $170.4(2)$ \\
\hline $\mathrm{N}(13)-\mathrm{Cu}(2)-\mathrm{N}(4)$ & $85.6(3)$ \\
\hline$N(5)-P(5)-O(10)$ & $110.7(3)$ \\
\hline$N(5)-P(5)-O(9)$ & $107.7(3)$ \\
\hline$O(10)-P(5)-O(9)$ & $100.5(3)$ \\
\hline$N(5)-P(5)-N(4)$ & $116.2(4)$ \\
\hline $\mathrm{O}(10)-\mathrm{P}(5)-\mathrm{N}(4)$ & $109.4(3)$ \\
\hline $\mathrm{O}(9)-\mathrm{P}(5)-\mathrm{N}(4)$ & $111.2(3)$ \\
\hline$N(5)-P(6)-N(6)$ & $116.6(4)$ \\
\hline$N(5)-P(6)-O(12)$ & $108.7(3)$ \\
\hline$N(6)-P(6)-O(12)$ & $114.2(3)$ \\
\hline$N(5)-P(6)-O(11)$ & $113.2(3)$ \\
\hline$N(6)-P(6)-O(11)$ & $107.8(3)$ \\
\hline $\mathrm{O}(12)-\mathrm{P}(6)-\mathrm{O}(11)$ & $94.2(3)$ \\
\hline$N(6)-P(4)-O(8)$ & $107.2(3)$ \\
\hline$N(6)-P(4)-O(7)$ & $109.7(3)$ \\
\hline $\mathrm{O}(8)-\mathrm{P}(4)-\mathrm{O}(7)$ & $100.1(3)$ \\
\hline$N(6)-P(4)-N(4)$ & $118.3(4)$ \\
\hline $\mathrm{O}(8)-\mathrm{P}(4)-\mathrm{N}(4)$ & $112.1(3)$ \\
\hline $\mathrm{O}(7)-\mathrm{P}(4)-\mathrm{N}(4)$ & $107.9(3)$ \\
\hline$N(2)-P(1)-O(1)$ & $107.1(3)$ \\
\hline $\mathrm{N}(2)-\mathrm{P}(1)-\mathrm{N}(1)$ & $117.7(3)$ \\
\hline $\mathrm{O}(1)-\mathrm{P}(1)-\mathrm{N}(1)$ & $111.1(3)$ \\
\hline$N(2)-P(1)-O(2)$ & $112.5(3)$ \\
\hline
\end{tabular}




\begin{tabular}{|c|c|}
\hline $\mathrm{O}(1)-\mathrm{P}(1)-\mathrm{O}(2)$ & $97.7(3)$ \\
\hline $\mathrm{N}(1)-\mathrm{P}(1)-\mathrm{O}(2)$ & $108.8(3)$ \\
\hline$O(4)-P(2)-O(3)$ & $99.7(3)$ \\
\hline $\mathrm{O}(4)-\mathrm{P}(2)-\mathrm{N}(3)$ & $112.3(3)$ \\
\hline $\mathrm{O}(3)-\mathrm{P}(2)-\mathrm{N}(3)$ & $110.2(3)$ \\
\hline $\mathrm{O}(4)-\mathrm{P}(2)-\mathrm{N}(2)$ & $104.7(3)$ \\
\hline $\mathrm{O}(3)-\mathrm{P}(2)-\mathrm{N}(2)$ & $110.4(3)$ \\
\hline $\mathrm{N}(3)-\mathrm{P}(2)-\mathrm{N}(2)$ & $117.9(4)$ \\
\hline$N(3)-P(3)-O(5)$ & $113.8(3)$ \\
\hline $\mathrm{N}(3)-\mathrm{P}(3)-\mathrm{O}(6)$ & $113.5(3)$ \\
\hline$O(5)-P(3)-O(6)$ & $99.9(3)$ \\
\hline $\mathrm{N}(3)-\mathrm{P}(3)-\mathrm{N}(1)$ & $117.1(3)$ \\
\hline$O(5)-P(3)-N(1)$ & $102.4(3)$ \\
\hline $\mathrm{O}(6)-\mathrm{P}(3)-\mathrm{N}(1)$ & $108.2(3)$ \\
\hline$C(16)-C(15)-C(14)$ & $120.0(9)$ \\
\hline$C(16)-C(15)-C(18)$ & $121.1(9)$ \\
\hline$C(14)-C(15)-C(18)$ & $118.9(9)$ \\
\hline $\mathrm{N}(11)-\mathrm{C}(13)-\mathrm{C}(14)$ & $128.6(8)$ \\
\hline $\mathrm{N}(11)-\mathrm{C}(13)-\mathrm{O}(3)$ & $117.5(7)$ \\
\hline$C(14)-C(13)-O(3)$ & $113.9(8)$ \\
\hline$C(15)-C(14)-C(13)$ & $114.6(9)$ \\
\hline$C(15)-C(16)-C(17)$ & $118.3(9)$ \\
\hline $\mathrm{N}(11)-\mathrm{C}(17)-\mathrm{C}(16)$ & $124.2(9)$ \\
\hline $\mathrm{N}(12)-\mathrm{C}(19)-\mathrm{C}(20)$ & $126.3(9)$ \\
\hline $\mathrm{N}(12)-\mathrm{C}(19)-\mathrm{O}(4)$ & $110.2(7)$ \\
\hline$C(20)-C(19)-O(4)$ & $123.5(8)$ \\
\hline $\mathrm{C}(19)-\mathrm{N}(12)-\mathrm{C}(23)$ & $115.0(8)$ \\
\hline$C(21)-C(22)-C(23)$ & $121.1(10)$ \\
\hline$C(22)-C(21)-C(20)$ & $116.7(9)$ \\
\hline$C(22)-C(21)-C(24)$ & $122.4(9)$ \\
\hline$C(20)-C(21)-C(24)$ & $120.9(9)$ \\
\hline $\mathrm{N}(12)-\mathrm{C}(23)-\mathrm{C}(22)$ & $122.9(10)$ \\
\hline $\mathrm{N}(10)-\mathrm{C}(35)-\mathrm{C}(34)$ & $123.4(9)$ \\
\hline $\mathrm{N}(10)-\mathrm{C}(31)-\mathrm{C}(32)$ & $126.0(8)$ \\
\hline $\mathrm{N}(10)-\mathrm{C}(31)-\mathrm{O}(6)$ & $116.6(7)$ \\
\hline$C(32)-C(31)-O(6)$ & $117.4(8)$ \\
\hline$C(31)-C(32)-C(33)$ & $118.4(8)$ \\
\hline$C(35)-C(34)-C(33)$ & $120.8(8)$ \\
\hline$C(32)-C(33)-C(34)$ & $116.5(8)$ \\
\hline$C(32)-C(33)-C(36)$ & $121.3(9)$ \\
\hline$C(34)-C(33)-C(36)$ & $122.2(9)$ \\
\hline$C(8)-C(9)-C(10)$ & $116.4(8)$ \\
\hline$C(8)-C(9)-C(12)$ & $121.8(8)$ \\
\hline$C(10)-C(9)-C(12)$ & $121.7(8)$ \\
\hline$C(11)-C(10)-C(9)$ & $120.3(8)$ \\
\hline $\mathrm{C}(10)-\mathrm{C}(11)-\mathrm{N}(8)$ & $123.6(8)$ \\
\hline $\mathrm{N}(8)-\mathrm{C}(7)-\mathrm{C}(8)$ & $124.7(8)$ \\
\hline$N(8)-C(7)-O(2)$ & $119.0(7)$ \\
\hline$C(8)-C(7)-O(2)$ & $116.2(7)$ \\
\hline$N(9)-C(25)-C(26)$ & $127.1(8)$ \\
\hline$N(9)-C(25)-O(5)$ & $116.4(8)$ \\
\hline$C(26)-C(25)-O(5)$ & $116.4(8)$ \\
\hline$C(25)-C(26)-C(27)$ & $119.1(8)$ \\
\hline$C(28)-C(27)-C(26)$ & $114.4(9)$ \\
\hline$C(28)-C(27)-C(30)$ & $124.9(9)$ \\
\hline$C(26)-C(27)-C(30)$ & $120.8(9)$ \\
\hline$C(29)-C(28)-C(27)$ & $122.3(9)$ \\
\hline$N(9)-C(29)-C(28)$ & $123.4(9)$ \\
\hline $\mathrm{N}(19)-\mathrm{C}(78)-\mathrm{C}(77)$ & $125.6(8)$ \\
\hline $\mathrm{O}(13)-\mathrm{C}(73)-\mathrm{N}(19)$ & $119.6(7)$ \\
\hline$O(13)-C(73)-C(74)$ & $122.6(8)$ \\
\hline $\mathrm{N}(19)-\mathrm{C}(73)-\mathrm{C}(74)$ & $117.8(8)$ \\
\hline$C(75)-C(74)-C(73)$ & $123.5(8)$ \\
\hline $\mathrm{C}(78)-\mathrm{C}(77)-\mathrm{C}(75)$ & $117.9(8)$ \\
\hline$C(74)-C(75)-C(77)$ & $116.7(8)$ \\
\hline$C(74)-C(75)-C(76)$ & $124.2(8)$ \\
\hline$C(77)-C(75)-C(76)$ & $119.1(7)$ \\
\hline $\mathrm{N}(14)-\mathrm{C}(47)-\mathrm{C}(46)$ & $123.7(9)$ \\
\hline
\end{tabular}




\begin{tabular}{|c|c|}
\hline$C(47)-C(46)-C(45)$ & $120.2(9)$ \\
\hline$C(44)-C(45)-C(46)$ & $116.6(8)$ \\
\hline$C(44)-C(45)-C(48)$ & $121.4(9)$ \\
\hline$C(46)-C(45)-C(48)$ & $122.0(8)$ \\
\hline$C(45)-C(44)-C(43)$ & $119.0(9)$ \\
\hline$N(14)-C(43)-O(8)$ & $119.4(8)$ \\
\hline$N(14)-C(43)-C(44)$ & $124.5(9)$ \\
\hline $\mathrm{O}(8)-\mathrm{C}(43)-\mathrm{C}(44)$ & $116.1(8)$ \\
\hline $\mathrm{N}(18)-\mathrm{C}(67)-\mathrm{C}(68)$ & $127.9(9)$ \\
\hline$N(18)-C(67)-O(12)$ & $116.3(7)$ \\
\hline$C(68)-C(67)-O(12)$ & $115.7(9)$ \\
\hline$C(67)-C(68)-C(69)$ & $117.1(9)$ \\
\hline$C(68)-C(69)-C(70)$ & $116.3(8)$ \\
\hline$C(68)-C(69)-C(72)$ & $121.4(9)$ \\
\hline$C(70)-C(69)-C(72)$ & $122.3(8)$ \\
\hline$C(71)-C(70)-C(69)$ & $121.2(8)$ \\
\hline $\mathrm{C}(70)-\mathrm{C}(71)-\mathrm{N}(18)$ & $122.8(9)$ \\
\hline$N(17)-C(61)-C(62)$ & $126.9(8)$ \\
\hline$N(17)-C(61)-O(11)$ & $117.5(8)$ \\
\hline$C(62)-C(61)-O(11)$ & $115.6(8)$ \\
\hline$C(61)-C(62)-C(63)$ & $116.9(9)$ \\
\hline$C(64)-C(63)-C(62)$ & $117.2(8)$ \\
\hline$C(64)-C(63)-C(66)$ & $123.4(8)$ \\
\hline$C(62)-C(63)-C(66)$ & $119.4(9)$ \\
\hline$C(63)-C(64)-C(65)$ & $120.4(8)$ \\
\hline $\mathrm{N}(17)-\mathrm{C}(65)-\mathrm{C}(64)$ & $123.2(9)$ \\
\hline$N(13)-C(37)-C(38)$ & $127.2(8)$ \\
\hline $\mathrm{N}(13)-\mathrm{C}(37)-\mathrm{O}(7)$ & $117.0(7)$ \\
\hline$C(38)-C(37)-O(7)$ & $115.7(8)$ \\
\hline$C(37)-C(38)-C(39)$ & $116.8(9)$ \\
\hline$C(40)-C(39)-C(38)$ & $117.5(8)$ \\
\hline$C(40)-C(39)-C(42)$ & $123.3(8)$ \\
\hline$C(38)-C(39)-C(42)$ & $119.3(9)$ \\
\hline$C(39)-C(40)-C(41)$ & $121.9(8)$ \\
\hline $\mathrm{C}(40)-\mathrm{C}(41)-\mathrm{N}(13)$ & $121.7(9)$ \\
\hline$N(15)-C(49)-C(50)$ & $128.0(8)$ \\
\hline $\mathrm{N}(15)-\mathrm{C}(49)-\mathrm{O}(9)$ & $118.3(7)$ \\
\hline$C(50)-C(49)-O(9)$ & $113.7(8)$ \\
\hline$C(51)-C(50)-C(49)$ & $116.5(8)$ \\
\hline$C(50)-C(51)-C(52)$ & $117.8(8)$ \\
\hline$C(50)-C(51)-C(54)$ & $119.8(8)$ \\
\hline$C(52)-C(51)-C(54)$ & $122.4(8)$ \\
\hline$C(53)-C(52)-C(51)$ & $119.5(8)$ \\
\hline$N(15)-C(53)-C(52)$ & $123.7(8)$ \\
\hline$C(5)-C(4)-C(3)$ & $118.7(8)$ \\
\hline$N(16)-C(55)-C(56)$ & $126.7(7)$ \\
\hline$N(16)-C(55)-O(10)$ & $114.3(7)$ \\
\hline$C(56)-C(55)-O(10)$ & $118.9(7)$ \\
\hline$N(7)-C(1)-C(2)$ & $125.1(8)$ \\
\hline$N(7)-C(1)-O(1)$ & $117.2(7)$ \\
\hline$C(2)-C(1)-O(1)$ & $117.6(8)$ \\
\hline$C(1)-C(2)-C(3)$ & $117.4(8)$ \\
\hline$N(16)-C(59)-C(58)$ & $123.0(8)$ \\
\hline$C(55)-C(56)-C(57)$ & $118.6(8)$ \\
\hline$C(56)-C(57)-C(58)$ & $115.6(8)$ \\
\hline$C(56)-C(57)-C(60)$ & $121.0(8)$ \\
\hline$C(58)-C(57)-C(60)$ & $123.5(8)$ \\
\hline$C(7)-C(8)-C(9)$ & $119.6(8)$ \\
\hline$C(2)-C(3)-C(4)$ & $118.5(8)$ \\
\hline$C(2)-C(3)-C(6)$ & $120.5(8)$ \\
\hline$C(4)-C(3)-C(6)$ & $121.0(8)$ \\
\hline$N(7)-C(5)-C(4)$ & $123.7(8)$ \\
\hline$C(59)-C(58)-C(57)$ & $121.6(8)$ \\
\hline$N(90)-C(90)-C(91)$ & $178.7(16)$ \\
\hline$P(1)-N(1)-P(3)$ & $121.1(4)$ \\
\hline $\mathrm{P}(1)-\mathrm{N}(1)-\mathrm{Cu}(1)$ & $113.0(3)$ \\
\hline $\mathrm{P}(3)-\mathrm{N}(1)-\mathrm{Cu}(1)$ & $122.9(3)$ \\
\hline$P(3)-N(3)-P(2)$ & $122.1(4)$ \\
\hline
\end{tabular}




\begin{tabular}{|c|c|}
\hline$P(1)-N(2)-P(2)$ & $122.3(4)$ \\
\hline$P(4)-N(4)-P(5)$ & $120.3(4)$ \\
\hline $\mathrm{P}(4)-\mathrm{N}(4)-\mathrm{Cu}(2)$ & $115.4(4)$ \\
\hline $\mathrm{P}(5)-\mathrm{N}(4)-\mathrm{Cu}(2)$ & $122.8(4)$ \\
\hline$P(4)-N(6)-P(6)$ & $122.2(4)$ \\
\hline$P(5)-N(5)-P(6)$ & $123.3(4)$ \\
\hline $\mathrm{C}(61)-\mathrm{N}(17)-\mathrm{C}(65)$ & $115.3(8)$ \\
\hline $\mathrm{C}(67)-\mathrm{N}(18)-\mathrm{C}(71)$ & $114.6(7)$ \\
\hline $\mathrm{C}(55)-\mathrm{N}(16)-\mathrm{C}(59)$ & $114.6(7)$ \\
\hline $\mathrm{C}(47)-\mathrm{N}(14)-\mathrm{C}(43)$ & $115.9(7)$ \\
\hline $\mathrm{C}(37)-\mathrm{N}(13)-\mathrm{C}(41)$ & $114.9(7)$ \\
\hline $\mathrm{C}(37)-\mathrm{N}(13)-\mathrm{Cu}(2)$ & $124.2(5)$ \\
\hline $\mathrm{C}(41)-\mathrm{N}(13)-\mathrm{Cu}(2)$ & $120.6(6)$ \\
\hline $\mathrm{C}(78)-\mathrm{N}(19)-\mathrm{C}(73)$ & $118.3(7)$ \\
\hline $\mathrm{C}(78)-\mathrm{N}(19)-\mathrm{Cu}(1)$ & $119.5(6)$ \\
\hline $\mathrm{C}(73)-\mathrm{N}(19)-\mathrm{Cu}(1)$ & $121.8(5)$ \\
\hline$C(5)-N(7)-C(1)$ & $116.6(7)$ \\
\hline $\mathrm{C}(5)-\mathrm{N}(7)-\mathrm{Cu}(1)$ & $115.7(6)$ \\
\hline $\mathrm{C}(1)-\mathrm{N}(7)-\mathrm{Cu}(1)$ & $127.2(6)$ \\
\hline$C(7)-N(8)-C(11)$ & $115.1(7)$ \\
\hline $\mathrm{C}(7)-\mathrm{N}(8)-\mathrm{Cu}(1)$ & $124.1(5)$ \\
\hline $\mathrm{C}(11)-\mathrm{N}(8)-\mathrm{Cu}(1)$ & $120.8(6)$ \\
\hline $\mathrm{C}(31)-\mathrm{N}(10)-\mathrm{C}(35)$ & $114.8(7)$ \\
\hline $\mathrm{C}(13)-\mathrm{N}(11)-\mathrm{C}(17)$ & $114.4(7)$ \\
\hline $\mathrm{C}(25)-\mathrm{N}(9)-\mathrm{C}(29)$ & $113.8(8)$ \\
\hline$C(19)-C(20)-C(21)$ & $117.9(9)$ \\
\hline $\mathrm{C}(49)-\mathrm{N}(15)-\mathrm{C}(53)$ & $114.4(7)$ \\
\hline $\mathrm{Cu}(1)-\mathrm{O}(14)-\mathrm{Cu}(2)$ & $126.8(3)$ \\
\hline $\mathrm{C}(73)-\mathrm{O}(13)-\mathrm{Cu}(2)$ & $127.3(5)$ \\
\hline$C(49)-O(9)-P(5)$ & $130.5(5)$ \\
\hline$C(55)-O(10)-P(5)$ & $120.8(5)$ \\
\hline $\mathrm{C}(67)-\mathrm{O}(12)-\mathrm{P}(6)$ & $121.1(5)$ \\
\hline $\mathrm{C}(61)-\mathrm{O}(11)-\mathrm{P}(6)$ & $120.5(5)$ \\
\hline $\mathrm{C}(37)-\mathrm{O}(7)-\mathrm{P}(4)$ & $123.3(5)$ \\
\hline$C(43)-O(8)-P(4)$ & $126.1(5)$ \\
\hline $\mathrm{C}(1)-\mathrm{O}(1)-\mathrm{P}(1)$ & $121.6(5)$ \\
\hline$C(13)-O(3)-P(2)$ & $123.0(5)$ \\
\hline $\mathrm{C}(19)-\mathrm{O}(4)-\mathrm{P}(2)$ & $130.0(5)$ \\
\hline$C(31)-O(6)-P(3)$ & $122.7(5)$ \\
\hline$C(25)-O(5)-P(3)$ & $127.2(5)$ \\
\hline $\mathrm{C}(7)-\mathrm{O}(2)-\mathrm{P}(1)$ & $126.2(5)$ \\
\hline$F(6)-P(7)-F(3)$ & $179.4(4)$ \\
\hline$F(6)-P(7)-F(4)$ & $88.9(4)$ \\
\hline$F(3)-P(7)-F(4)$ & $90.9(4)$ \\
\hline$F(6)-P(7)-F(5)$ & $89.9(3)$ \\
\hline$F(3)-P(7)-F(5)$ & $89.5(4)$ \\
\hline$F(4)-P(7)-F(5)$ & $90.4(3)$ \\
\hline$F(6)-P(7)-F(1)$ & $90.8(4)$ \\
\hline$F(3)-P(7)-F(1)$ & $89.8(4)$ \\
\hline$F(4)-P(7)-F(1)$ & $89.9(3)$ \\
\hline$F(5)-P(7)-F(1)$ & $179.2(4)$ \\
\hline$F(6)-P(7)-F(2)$ & $89.9(4)$ \\
\hline$F(3)-P(7)-F(2)$ & $90.3(4)$ \\
\hline$F(4)-P(7)-F(2)$ & $178.8(4)$ \\
\hline$F(5)-P(7)-F(2)$ & $90.0(3)$ \\
\hline$F(1)-P(7)-F(2)$ & $89.7(3)$ \\
\hline$F(7 B)-P(8)-F(11 A)$ & $123.0(8)$ \\
\hline$F(7 B)-P(8)-F(10 A)$ & $139.8(7)$ \\
\hline$F(11 A)-P(8)-F(10 A)$ & $96.5(6)$ \\
\hline$F(7 B)-P(8)-F(8 B)$ & $96.7(8)$ \\
\hline$F(11 A)-P(8)-F(8 B)$ & $122.3(8)$ \\
\hline$F(10 A)-P(8)-F(8 B)$ & $63.6(7)$ \\
\hline$F(7 B)-P(8)-F(12 B)$ & $95.3(6)$ \\
\hline$F(11 A)-P(8)-F(12 B)$ & $49.4(7)$ \\
\hline$F(10 A)-P(8)-F(12 B)$ & $118.4(7)$ \\
\hline $\mathrm{F}(8 \mathrm{~B})-\mathrm{P}(8)-\mathrm{F}(12 \mathrm{~B})$ & $91.2(8)$ \\
\hline$F(7 B)-P(8)-F(12 A)$ & $92.9(7)$ \\
\hline$F(11 A)-P(8)-F(12 A)$ & $93.3(6)$ \\
\hline
\end{tabular}




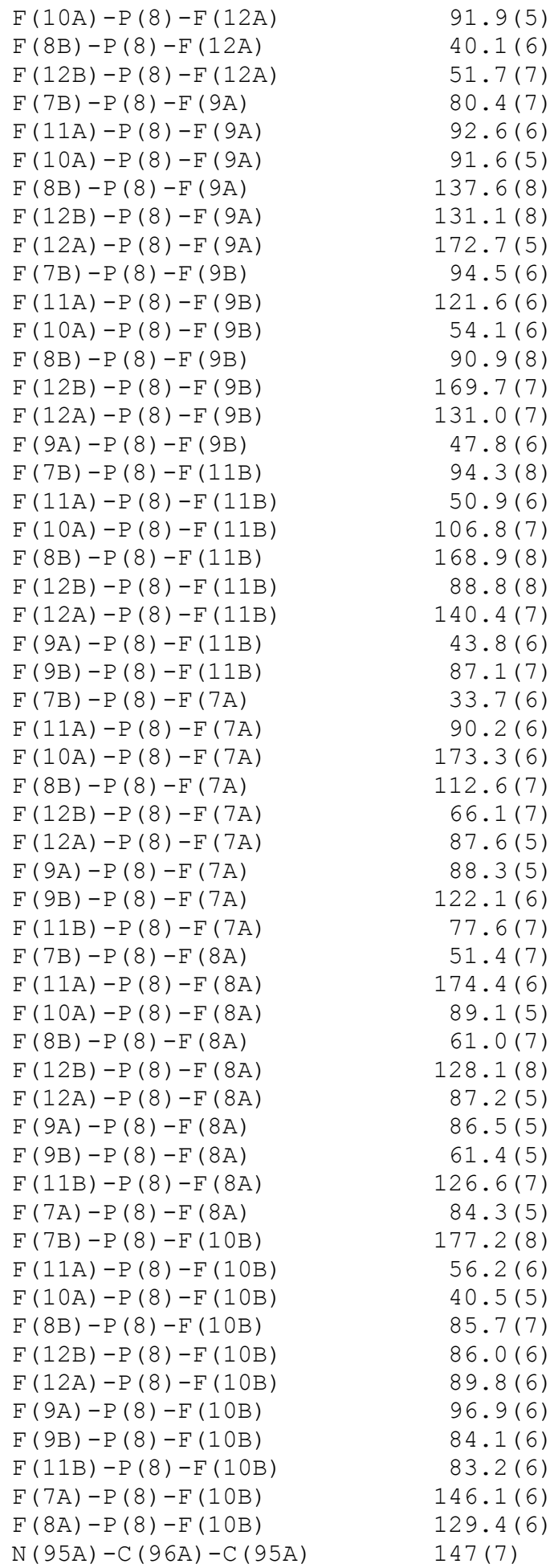

$91.9(5)$

$40.1(6)$

$51.7(7)$

$80.4(7)$

$92.6(6)$

$91.6(5)$

$137.6(8)$

$131.1(8)$

$172.7(5)$

$94.5(6)$

$121.6(6)$

$54.1(6)$

$90.9(8)$

$169.7(7)$

$131.0(7)$

$47.8(6)$

$94.3(8)$

$50.9(6)$

$106.8(7)$

$168.9(8)$

$88.8(8)$

$140.4(7)$

$43.8(6)$

$87.1(7)$

$33.7(6)$

$90.2(6)$

$173.3(6)$

$112.6(7)$

$66.1(7)$

$87.6(5)$

$88.3(5)$

$122.1(6)$

$77.6(7)$

$51.4(7)$

$174.4(6)$

$89.1(5)$

$61.0(7)$

$128.1(8)$

$87.2(5)$

$86.5(5)$

$61.4(5)$

$126.6(7)$

$84.3(5)$

$177.2(8)$

$56.2(6)$

$40.5(5)$

$85.7(7)$

$86.0(6)$

$89.8(6)$

$96.9(6)$

$84.1(6)$

$83.2(6)$

$146.1(6)$

$129.4(6)$

$147(7)$ 
Table 9. Anisotropic displacement parameters $\left(\AA^{2} \times 10^{3}\right)$ for $\left[\mathrm{Cu}_{2}(\mathrm{MeL})_{2}(\mu-\mathrm{OH})(\boldsymbol{\mu}-4-\mathrm{MeOpy})\right]\left(\mathrm{PF}_{6}\right)_{2} \cdot 2 \mathrm{CH}_{3} \mathrm{CN} \quad$ (5) .

The anisotropic displacement factor exponent takes the form: $-2 \pi^{2}\left[h^{2} a \star^{2} U^{11}+\ldots+2 h k a * b * U^{12}\right]$.

\begin{tabular}{|c|c|c|c|c|c|c|}
\hline & $\mathrm{U}^{11}$ & $\mathrm{U}^{22}$ & $\mathrm{U}^{33}$ & $\mathrm{U}^{23}$ & $\mathrm{U}^{13}$ & $\mathrm{U}^{12}$ \\
\hline C (15) & $43(7)$ & $27(6)$ & $43(7)$ & $-7(5)$ & $30(6)$ & $-2(5)$ \\
\hline$C(13)$ & $33(6)$ & $8(4)$ & $25(6)$ & $-6(4)$ & $15(5)$ & $-3(4)$ \\
\hline$C(14)$ & $21(6)$ & $16(5)$ & $41(6)$ & $-8(4)$ & $9(5)$ & $-4(4)$ \\
\hline$C(16)$ & $35(7)$ & $28(5)$ & $36(6)$ & $-8(5)$ & $15(5)$ & $-1(5)$ \\
\hline C (18) & $35(7)$ & $53(7)$ & $56(7)$ & $-8(6)$ & $29(6)$ & $5(5)$ \\
\hline C (17) & $31(6)$ & $33(6)$ & $21(6)$ & $-8(5)$ & $8(5)$ & $3(5)$ \\
\hline C (19) & $25(6)$ & $29(6)$ & $21(6)$ & $0(5)$ & $7(5)$ & $0(5)$ \\
\hline$N(12)$ & $38(6)$ & $36(5)$ & $47(6)$ & $7(5)$ & $21(5)$ & $-4(4)$ \\
\hline$C(22)$ & $40(7)$ & $48(7)$ & $29(7)$ & $-10(5)$ & $12(5)$ & $-9(6)$ \\
\hline$C(21)$ & $36(7)$ & $40(6)$ & $37(7)$ & $-6(5)$ & $19(6)$ & $-4(5)$ \\
\hline C (23) & $60(9)$ & $65(8)$ & $26(7)$ & $-13(6)$ & $11(6)$ & $-11(7)$ \\
\hline C (35) & $37(7)$ & $22(5)$ & $32(6)$ & $-3(5)$ & $12(5)$ & $-2(5)$ \\
\hline C (31) & $24(6)$ & $16(5)$ & $20(5)$ & $-8(4)$ & $3(4)$ & $-9(4)$ \\
\hline$C(32)$ & $19(5)$ & $15(5)$ & $32(6)$ & $-5(4)$ & $8(5)$ & $6(4)$ \\
\hline C (34) & $40(6)$ & $29(6)$ & $22(6)$ & $-3(5)$ & $18(5)$ & $-8(5)$ \\
\hline C (33) & $39(7)$ & $37(6)$ & $33(6)$ & $-20(5)$ & $21(5)$ & $-14(5)$ \\
\hline$C(36)$ & $55(8)$ & $56(7)$ & $56(8)$ & $-7(6)$ & $37(7)$ & $-4(6)$ \\
\hline$C(12)$ & $71(9)$ & $35(6)$ & $44(7)$ & $12(5)$ & $38(7)$ & $6(6)$ \\
\hline C (9) & $39(6)$ & $20(5)$ & $25(6)$ & $-3(4)$ & $23(5)$ & $-7(5)$ \\
\hline$C(10)$ & $32(6)$ & $22(5)$ & $36(6)$ & $1(5)$ & $21(5)$ & $-5(4)$ \\
\hline$C(11)$ & $23(6)$ & $14(5)$ & $37(6)$ & $-6(4)$ & $12(5)$ & $-5(4)$ \\
\hline$C(7)$ & $23(5)$ & $9(4)$ & $17(5)$ & $-5(4)$ & $12(4)$ & $-8(4)$ \\
\hline C (25) & $11(5)$ & $23(5)$ & $25(6)$ & $5(5)$ & $-2(4)$ & $2(4)$ \\
\hline$C(26)$ & $25(6)$ & $34(6)$ & $19(6)$ & $-4(4)$ & $12(5)$ & $1(5)$ \\
\hline C (27) & $13(5)$ & $33(6)$ & $32(6)$ & $7(5)$ & $2(5)$ & $0(4)$ \\
\hline$C(30)$ & $38(7)$ & $49(7)$ & $49(7)$ & $23(6)$ & $24(6)$ & $3(5)$ \\
\hline C (28) & $17(6)$ & $14(5)$ & $64(8)$ & $5(5)$ & $1(6)$ & $-4(4)$ \\
\hline C (29) & $44(8)$ & $29(6)$ & $57(8)$ & $-4(6)$ & $19(6)$ & $-3(5)$ \\
\hline$C(78)$ & $26(6)$ & $15(5)$ & $24(5)$ & $3(4)$ & $16(5)$ & $1(4)$ \\
\hline C (73) & $26(6)$ & $16(5)$ & $14(5)$ & $-5(4)$ & $6(4)$ & $-4(4)$ \\
\hline$C(74)$ & $35(6)$ & $12(5)$ & $23(5)$ & $-4(4)$ & $13(5)$ & $-3(4)$ \\
\hline C (77) & $14(5)$ & $29(5)$ & $19(5)$ & $-7(4)$ & $2(4)$ & $-5(4)$ \\
\hline$C(75)$ & $23(5)$ & $16(5)$ & $27(6)$ & $-5(4)$ & $13(5)$ & $-5(4)$ \\
\hline$C(76)$ & $37(7)$ & $28(5)$ & $38(6)$ & $1(5)$ & $22(5)$ & $3(5)$ \\
\hline C (47) & $44(7)$ & $25(5)$ & $24(6)$ & $-1(4)$ & $25(5)$ & $3(5)$ \\
\hline$C(46)$ & $37(6)$ & $26(5)$ & $29(6)$ & $5(5)$ & $23(5)$ & $12(5)$ \\
\hline C ( 45$)$ & $53(7)$ & $11(5)$ & $35(6)$ & $-2(4)$ & $28(6)$ & $1(5)$ \\
\hline C (48) & $71(8)$ & $23(5)$ & $61(8)$ & $-3(5)$ & $53(7)$ & $3(5)$ \\
\hline C ( 44$)$ & $54(7)$ & $17(5)$ & $31(6)$ & $-6(4)$ & $25(6)$ & $-10(5)$ \\
\hline C (43) & $35(7)$ & $30(6)$ & $23(6)$ & $4(5)$ & $14(5)$ & $-1(5)$ \\
\hline$C(67)$ & $32(6)$ & $19(5)$ & $27(6)$ & $2(4)$ & $4(5)$ & $-6(5)$ \\
\hline C (68) & $23(6)$ & $32(6)$ & $37(6)$ & $-1(5)$ & $7(5)$ & $-4(5)$ \\
\hline C (69) & $42(7)$ & $20(5)$ & $18(5)$ & $3(4)$ & $10(5)$ & $-5(5)$ \\
\hline$C(70)$ & $40(7)$ & $15(5)$ & $35(6)$ & $-5(5)$ & $9(5)$ & $0(5)$ \\
\hline$C(71)$ & $25(6)$ & $33(6)$ & $21(6)$ & $10(5)$ & $5(5)$ & $10(5)$ \\
\hline$C(72)$ & $46(7)$ & $31(6)$ & $38(7)$ & $9(5)$ & $10(6)$ & $-14(5)$ \\
\hline$C(6)$ & $52(7)$ & $14(5)$ & $33(6)$ & $-7(4)$ & $27(6)$ & $3(5)$ \\
\hline C (61) & $27(6)$ & $24(5)$ & $31(6)$ & $-4(5)$ & $18(5)$ & $2(5)$ \\
\hline C (62) & $21(6)$ & $33(6)$ & $28(6)$ & $-7(5)$ & $4(5)$ & $7(5)$ \\
\hline C (63) & $42(7)$ & $9(5)$ & $32(6)$ & $6(4)$ & $7(5)$ & $11(5)$ \\
\hline$C(66)$ & $33(7)$ & $18(5)$ & $70(8)$ & $2(5)$ & $4(6)$ & $5(5)$ \\
\hline$C(64)$ & $34(7)$ & $17(5)$ & $52(7)$ & $5(5)$ & $21(6)$ & $7(5)$ \\
\hline$C(65)$ & $27(6)$ & $33(6)$ & $27(6)$ & $-8(5)$ & $8(5)$ & $-10(5)$ \\
\hline C (37) & $24(6)$ & $12(5)$ & $30(6)$ & $10(4)$ & $19(5)$ & $10(4)$ \\
\hline C (38) & $28(6)$ & $30(6)$ & $28(6)$ & $5(5)$ & $17(5)$ & $9(5)$ \\
\hline C (39) & $28(6)$ & $17(5)$ & $31(6)$ & $14(4)$ & $16(5)$ & $15(4)$ \\
\hline$C(42)$ & $40(7)$ & $22(5)$ & $47(7)$ & $10(5)$ & $24(6)$ & $10(5)$ \\
\hline$C(40)$ & $36(7)$ & $22(5)$ & $34(6)$ & $8(5)$ & $24(5)$ & $6(5)$ \\
\hline
\end{tabular}




\begin{tabular}{|c|c|c|c|c|c|c|}
\hline C (41) & $30(6)$ & $22(5)$ & $31(6)$ & $-2(5)$ & $12(5)$ & $-7(5)$ \\
\hline C (49) & $17(5)$ & $22(5)$ & $18(5)$ & $2(4)$ & $3(4)$ & $-6(4)$ \\
\hline$C(50)$ & $33(6)$ & $16(5)$ & $22(5)$ & $-2(4)$ & $14(5)$ & $1(4)$ \\
\hline C (51) & $31(6)$ & $14(5)$ & $18(5)$ & $-1(4)$ & $7(5)$ & $-7(4)$ \\
\hline$C(54)$ & $48(7)$ & $27(6)$ & $35(6)$ & $-11(5)$ & $12(6)$ & $-6(5)$ \\
\hline$C(52)$ & $30(6)$ & $33(6)$ & $30(6)$ & $-4(5)$ & $6(5)$ & $-20(5)$ \\
\hline C (53) & $16(6)$ & $40(6)$ & $27(6)$ & $-8(5)$ & $13(5)$ & $-4(5)$ \\
\hline$C(4)$ & $20(5)$ & $18(5)$ & $22(5)$ & $-1(4)$ & $8(4)$ & $-3(4)$ \\
\hline$C(55)$ & $29(6)$ & $10(5)$ & $17(5)$ & $-1(4)$ & $3(5)$ & $2(4)$ \\
\hline$C(1)$ & $21(6)$ & $28(5)$ & $10(5)$ & $3(4)$ & $6(4)$ & $-2(5)$ \\
\hline C (2) & $34(6)$ & $15(5)$ & $20(5)$ & $4(4)$ & $18(5)$ & $6(4)$ \\
\hline C (59) & $35(6)$ & $18(5)$ & $38(6)$ & $1(5)$ & $19(5)$ & $4(4)$ \\
\hline$C(56)$ & $32(6)$ & $32(6)$ & $17(5)$ & $-3(4)$ & $13(5)$ & $-5(5)$ \\
\hline$C(57)$ & $41(7)$ & $16(5)$ & $20(6)$ & $5(4)$ & $10(5)$ & $-2(4)$ \\
\hline$C(8)$ & $35(6)$ & $16(5)$ & $19(5)$ & $5(4)$ & $10(5)$ & $-2(4)$ \\
\hline$C(3)$ & $36(6)$ & $22(5)$ & $18(5)$ & $1(4)$ & $16(5)$ & $-3(5)$ \\
\hline C (5) & $28(6)$ & $13(5)$ & $26(6)$ & $2(4)$ & $13(5)$ & $5(4)$ \\
\hline$C(60)$ & $58(8)$ & $28(6)$ & $58(8)$ & $12(5)$ & $25(7)$ & $-8(5)$ \\
\hline$C(58)$ & $46(7)$ & $16(5)$ & $29(6)$ & $5(5)$ & $8(5)$ & $4(5)$ \\
\hline$C(24)$ & $67(9)$ & $30(6)$ & $60(8)$ & $3(6)$ & $33(7)$ & $3(6)$ \\
\hline C (90) & $73(10)$ & $52(8)$ & $55(8)$ & $-7(7)$ & $38(8)$ & $-12(7)$ \\
\hline C (91) & $124(14)$ & $42(8)$ & $189(18)$ & $25(10)$ & $96(14)$ & $-10(8)$ \\
\hline $\mathrm{Cu}(1)$ & $22(1)$ & $16(1)$ & $21(1)$ & $1(1)$ & $12(1)$ & $2(1)$ \\
\hline $\mathrm{Cu}(2)$ & $22(1)$ & $15(1)$ & $27(1)$ & $4(1)$ & $13(1)$ & $3(1)$ \\
\hline $\mathrm{F}(1)$ & $96(6)$ & $32(3)$ & $58(4)$ & $0(3)$ & $17(4)$ & $-19(3)$ \\
\hline$F(2)$ & $83(5)$ & $56(4)$ & $48(4)$ & $-14(3)$ & $30(4)$ & $-10(3)$ \\
\hline$F(3)$ & $50(5)$ & $68(4)$ & $78(5)$ & $-6(4)$ & $-5(4)$ & $5(4)$ \\
\hline$F(4)$ & 105 (6) & $44(4)$ & $41(4)$ & $-2(3)$ & $36(4)$ & $-16(3)$ \\
\hline$F(5)$ & $79(5)$ & $36(3)$ & $63(4)$ & $-4(3)$ & $41(4)$ & $-14(3)$ \\
\hline$F(6)$ & $51(4)$ & $73(4)$ & $67(4)$ & $18(4)$ & $18(4)$ & $0(4)$ \\
\hline$F(7 A)$ & $110(11)$ & $70(9)$ & $58(8)$ & $-2(7)$ & $37(8)$ & $-13(7)$ \\
\hline$F(8 A)$ & $55(7)$ & $42(6)$ & $66(8)$ & $-13(5)$ & $26(6)$ & $12(5)$ \\
\hline$F(9 A)$ & $39(7)$ & $77(9)$ & $107(11)$ & $15(8)$ & $15(7)$ & $-26(6)$ \\
\hline$F(10 A)$ & $88(9)$ & $89(9)$ & $49(7)$ & $0(7)$ & $40(7)$ & $4(7)$ \\
\hline$F(11 A)$ & $119(11)$ & $19(5)$ & $123(11)$ & $-25(6)$ & $68(9)$ & $-4(6)$ \\
\hline$F(12 A)$ & $26(6)$ & $41(7)$ & $102(10)$ & $5(6)$ & $13(6)$ & $1(5)$ \\
\hline$F(7 B)$ & 77 (12) & $46(11)$ & $67(13)$ & $50(10)$ & $32(10)$ & $12(9)$ \\
\hline$F(8 B)$ & $78(14)$ & $62(12)$ & $86(13)$ & $-5(10)$ & $9(11)$ & $-39(11)$ \\
\hline$F(9 B)$ & 52 (11) & $60(10)$ & $55(11)$ & $28(8)$ & $46(9)$ & $26(8)$ \\
\hline$F(10 B)$ & $54(11)$ & $59(11)$ & $39(10)$ & $1(8)$ & $14(8)$ & $-5(8)$ \\
\hline$F(11 B)$ & $94(14)$ & $27(9)$ & $40(10)$ & $-3(8)$ & $-11(9)$ & $-8(9)$ \\
\hline$F(12 B)$ & $104(15)$ & $139(17)$ & $126(15)$ & $2(13)$ & $100(13)$ & $26(12)$ \\
\hline $\mathrm{N}(1)$ & $21(4)$ & $5(3)$ & $21(4)$ & $2(3)$ & $7(3)$ & $0(3)$ \\
\hline $\mathrm{N}(3)$ & $23(5)$ & $9(4)$ & $34(5)$ & $-1(3)$ & $9(4)$ & $1(3)$ \\
\hline $\mathrm{N}(2)$ & $23(5)$ & $15(4)$ & $29(5)$ & $-1(3)$ & $13(4)$ & $1(3)$ \\
\hline $\mathrm{N}(4)$ & $17(4)$ & $9(4)$ & $29(4)$ & $2(3)$ & $12(4)$ & $3(3)$ \\
\hline$N(6)$ & $21(5)$ & $12(4)$ & $27(4)$ & $0(3)$ & $4(4)$ & $-4(3)$ \\
\hline N (5) & $28(5)$ & $8(4)$ & $33(5)$ & $1(3)$ & $17(4)$ & $0(3)$ \\
\hline $\mathrm{N}(17)$ & $22(5)$ & $18(4)$ & $41(5)$ & $4(4)$ & $10(4)$ & $1(4)$ \\
\hline$N(18)$ & $29(5)$ & $16(4)$ & $35(5)$ & $5(4)$ & $11(4)$ & $6(4)$ \\
\hline$N(16)$ & $25(5)$ & $19(4)$ & $33(5)$ & $-3(4)$ & $18(4)$ & $-5(3)$ \\
\hline$N(14)$ & $33(5)$ & $16(4)$ & $22(4)$ & $-6(3)$ & $14(4)$ & $-2(4)$ \\
\hline$N(13)$ & $28(5)$ & $18(4)$ & $21(4)$ & $2(3)$ & $13(4)$ & $1(4)$ \\
\hline$N(19)$ & $20(4)$ & $14(4)$ & $29(5)$ & $-3(3)$ & $13(4)$ & $-3(3)$ \\
\hline$N(7)$ & $24(5)$ & $19(4)$ & $19(4)$ & $1(3)$ & $12(4)$ & $-2(4)$ \\
\hline $\mathrm{N}(8)$ & $14(4)$ & $13(4)$ & $28(4)$ & $-2(3)$ & $10(4)$ & $-2(3)$ \\
\hline$N(10)$ & $27(5)$ & $17(4)$ & $33(5)$ & $-4(4)$ & $12(4)$ & $-3(4)$ \\
\hline $\mathrm{N}(11)$ & $16(4)$ & $31(4)$ & $23(5)$ & $-8(4)$ & $1(4)$ & $-2(4)$ \\
\hline$N(9)$ & $34(5)$ & $14(4)$ & $46(6)$ & $0(4)$ & $19(4)$ & $1(4)$ \\
\hline$C(20)$ & $43(7)$ & $29(6)$ & $39(7)$ & $9(5)$ & $17(6)$ & $-2(5)$ \\
\hline$N(15)$ & $26(5)$ & $23(4)$ & $31(5)$ & $-1(4)$ & $19(4)$ & $-3(4)$ \\
\hline$N(90)$ & $63(8)$ & $78(8)$ & $60(7)$ & $2(6)$ & $34(6)$ & $-7(6)$ \\
\hline$O(14)$ & $14(3)$ & $22(3)$ & $21(3)$ & $5(3)$ & $9(3)$ & $2(3)$ \\
\hline$O(13)$ & $34(4)$ & $16(3)$ & $42(4)$ & $1(3)$ & $26(4)$ & $3(3)$ \\
\hline$O(9)$ & $17(4)$ & $14(3)$ & $40(4)$ & $-8(3)$ & $14(3)$ & $-2(3)$ \\
\hline$O(10)$ & $29(4)$ & $12(3)$ & $21(3)$ & $-1(3)$ & $13(3)$ & $-5(3)$ \\
\hline$O(12)$ & $14(4)$ & $21(3)$ & $48(4)$ & $1(3)$ & $15(3)$ & $-6(3)$ \\
\hline$O(11)$ & $20(4)$ & $22(3)$ & $42(4)$ & $8(3)$ & $15(3)$ & $1(3)$ \\
\hline
\end{tabular}




\begin{tabular}{lllrrrr}
$O(7)$ & $30(4)$ & $17(3)$ & $23(4)$ & $9(3)$ & $8(3)$ & $4(3)$ \\
$O(8)$ & $22(4)$ & $15(3)$ & $32(4)$ & $-1(3)$ & $9(3)$ & $1(3)$ \\
$O(1)$ & $21(4)$ & $14(3)$ & $25(4)$ & $-1(3)$ & $10(3)$ & $0(3)$ \\
$O(3)$ & $18(4)$ & $27(3)$ & $30(4)$ & $-1(3)$ & $9(3)$ & $3(3)$ \\
$O(4)$ & $27(4)$ & $19(3)$ & $24(4)$ & $0(3)$ & $5(3)$ & $-3(3)$ \\
$O(6)$ & $30(4)$ & $15(3)$ & $20(4)$ & $-2(3)$ & $9(3)$ & $4(3)$ \\
$O(5)$ & $31(4)$ & $22(3)$ & $22(4)$ & $2(3)$ & $11(3)$ & $-6(3)$ \\
$O(2)$ & $29(4)$ & $13(3)$ & $20(3)$ & $5(3)$ & $12(3)$ & $9(3)$ \\
$\mathrm{P}(5)$ & $23(1)$ & $16(1)$ & $24(1)$ & $0(1)$ & $13(1)$ & $0(1)$ \\
$\mathrm{P}(6)$ & $20(1)$ & $17(1)$ & $33(2)$ & $0(1)$ & $10(1)$ & $0(1)$ \\
$\mathrm{P}(4)$ & $26(2)$ & $16(1)$ & $26(1)$ & $0(1)$ & $13(1)$ & $1(1)$ \\
$\mathrm{P}(1)$ & $26(2)$ & $17(1)$ & $20(1)$ & $4(1)$ & $11(1)$ & $1(1)$ \\
$\mathrm{P}(2)$ & $24(1)$ & $21(1)$ & $26(2)$ & $0(1)$ & $10(1)$ & $1(1)$ \\
$\mathrm{P}(3)$ & $23(2)$ & $17(1)$ & $22(1)$ & $3(1)$ & $10(1)$ & $1(1)$ \\
$\mathrm{P}(7)$ & $50(2)$ & $37(2)$ & $41(2)$ & $2(2)$ & $11(2)$ & $-8(2)$ \\
$\mathrm{P}(8)$ & $37(2)$ & $39(2)$ & $48(2)$ & $-14(2)$ & $26(2)$ & $-9(1)$ \\
& & & & & & \\
\hline
\end{tabular}

